\title{
Die großen wissenschaftlichen Leistungen von Stefan Vogel (1925-2015) Teil 6 (Schluss). Die Pilzmückenblumen
}

Anton Weber und Günter Gerlach

\begin{abstract}
In the late 1950s, Stefan Vogel provided evidence that tiny flies (Dipteras) act as regular pollinators of the trap flowers of Ceropegia, Apocynaceae-Asclepiadoideae, (micromyiophily). These studies laid the foundation for later, breathtaking discoveries, namely that flowers of several non-related families have adapted to the pollination by another group of tiny flies: fungus gnats (Mycetophilidae, Sciaridae and relatives). In particular, Vogel demonstrated that the respective plants (mainly dwellers of the dark, damp forest floor, with flowers positioned within in or slightly above the forest litter) have obtained morphological and olfactory characters of fungi in the course of evolution. Such "fungus mimetes" represent a then undescribed ecological subcategory of the myiophilous floral syndrome. They have evolved (at least) in four plant families: Aristolochiaceae (birthwort family), Araceae (aroids), Corsiaceae, and Orchidaceae (orchids). Perhaps the most spectacular examples are provided by Aristolochia arborea, with a perfect mushroom dummy placed at the entrance of the pitfall flower, and species of the orchid genus Dracula, in which the labellum imitates the head of a gilled mushroom.
\end{abstract}

\section{Zusammenfassung}

Nachdem Stefan Vogel in den späten 1950er-Jahren bewiesen hatte, dass bei den Kesselfallenblumen von Ceropegia (Apocynaceae-Asclepiadoideae) winzige Fliegen als Bestäuber agieren (Mikromyiophilie), konnte er zwanzig Jahre später eine weitere und ganz spezielle Fliegengruppe als Bestäuber ausmachen: Pilzmücken (Mycetophilidae, Sciaridae u.a.). Vogel konnte weiter zeigen, dass manche Blüten oder Blütenstände im Laufe der Evolution gestaltliche und olfaktorische Merkmale von Pilzen angenommen haben. Solche „Pilzmimeten“ haben sich in mindestens vier Verwandtschaftskreisen entwickelt: Aristolochiaceae (Osterluzeigewächse), Araceae (Aronstabgewächse), Corsiaceae und Orchideen. Als die vielleicht spektakulärsten Fälle sind zu nennen: Aristolochia arborea, mit einer vor dem Eingang der Kesselfalle stehenden Hutpilz-Attrappe, und die Arten der Gattung Dracula (Orchidaceae), deren Labellum den Hut eines Lamellen- oder Leistenpilzes imitiert.

\section{Einleitung}

Die Beschäftigung mit Blüten, die durch atypische Blütenbesucher bestäubt werden, geht bis in die Anfänge der wissenschaftlichen Forschungsarbeit von Stefan Vogel zurück: Auf seiner ersten großen Forschungsreise nach Südafrika (1950/51) begegnete er mehreren Arten der Gattung Ceropegia (Asclepiadaceae, heute Apocynaceae-Asclepoioideae). Die Blüten dieser Gattung faszinierten ihn deshalb, weil sie (a) nach dem Prinzip der Kesselfallen funktionieren, (b) offensichtlich dem wenig bekannten Syndrom der Fliegenbestäubung zuzuordnen waren, aber (c) offensichtlich nicht für Fliegen normaler Größe bestimmt waren. Allerdings konnte Vogel an den Naturstandorten keine Insekten auffinden, die als Bestäuber in Frage gekommen wären.

Zurück in Mainz, begann er sich genauer mit den Ceropegien zu befassen. Erstens, mit dem bis dahin unverstandenen Bau der Blüten, zweitens, mit den Produktionsstätten des Duftes (vgl. Teil 3 unserer Serie, Weber \& Steinecke 2017) und drittens, mit dem Mechanismus der Kesselfallen und den Insekten, die als Bestäuber zu qualifizieren waren. Vogel kam zum Schluss, dass ausnahmslos winzige Fliegen für die Bestäubung verantwortlich waren. Die Fliegen gehörten verschiedenen Familien an: Ceratopogonidae (Gnitzen oder Bartmücken), Chloropidae (Halmfliegen) und Milichiidae (Nistfliegen).

Später, in den 1970er-Jahren, entdeckte Vogel eine weitere Gruppe von Kleinfliegen, die eine wichtige Rolle als Bestäuber spielen: die Pilzmücken. Es handelt sich hier um eine Reihe nahe verwandter Familien, von denen die Mycetophiliden (die „Pilzliebenden“, Pilzmücken im engeren Sinn) und die Sciariden (von Griechisch skiá, „Schatten“; wegen der schwärzlichen Farbe im Deutschen als Trauermücken bezeichnet) die wichtigsten sind.

Zuerst erwähnt wurden die Pilzmücken und ihre Rolle als Bestäuber in einem Vortrag, den Vogel im Rahmen eines Festsymposiums zu Ehren 
des holländischen Blüten- und Ausbreitungsökologen L. VAN DER Pijl hielt (Vogel 1973). Hier referierte Vogel über seine Studien zur Bestäubung von Asarum caudatum (Aristolochiaceae) und Arisarum proboscideum (Araceae), die er im Botanischen Garten Mainz durchgeführt hatte. In einer Art Ausblick wurden auch kurz Aristolochia arborea, Masdevallia p.p. (Arten, die mittlerweile in die Gattung Dracula abgetrennt wurden) und Corsia uniflora (= Arachnitis uniflora) erwähnt.

Fünf Jahre später legt Vogel in einer in zwei Teilen veröffentlichten Arbeit (Vogel 1978a, b) ein Kompendium seiner Forschungen zu diesem Thema vor. Die Arbeit trägt den Titel „Pilzmückenblumen als Pilzmimeten“ - ein Meisterwerk botanischer Forschung und akribischer Spurensuche, und spannend zu lesen wie ein Krimi. In dieser Arbeit wird neben den vorhin genannten Taxa auch die Araceengattung Arisaema behandelt (Vogel 1978b). In Zusammenarbeit mit dem Zoologen Jochen Martens (Univ. Mainz) widmet er dieser Gattung mit tödlichen Kesselfallen später noch eine weitere bahnbrechende Stu- die (Vogel \& Martens 2000). Die genannten Werke sind auch online zugänglich und als kostenpflichtiges pdf downloadbar, siehe die am Ende zitierten Internetseiten.

\section{Pilzmücken, Pilzmückenblumen und ihre Lebensweise}

Die Pilzmücken sind eine artenreiche und taxonomisch schwierige Insektengruppe. Innerhalb der Fliegen (Diptera) gehören sie zu den Mücken (Nematocera) und hier zur Überfamilie der Sciaroidea mit mehreren Familien, von denen die Mycetophilidae und Sciaridae die wichtigsten sind. Bei den Mycetophilidae wurden bisher etwa 3000 Arten in etwa 150 Gattungen beschrieben, aber die Artenzahl ist mit Sicherheit weitaus höher. Die Familie ist nach der Gattung Mycetophila benannt, die allein etwa 750 Arten umfasst. Eine zweite Gattung trägt den bezeichnenden Namen Fungivora („die Pilzfressende").

Pilzmücken erkennt man als solche leicht an dem hochgewölbten Buckel und den langen Beinen. Es handelt sich um eine sehr alte Insekten-

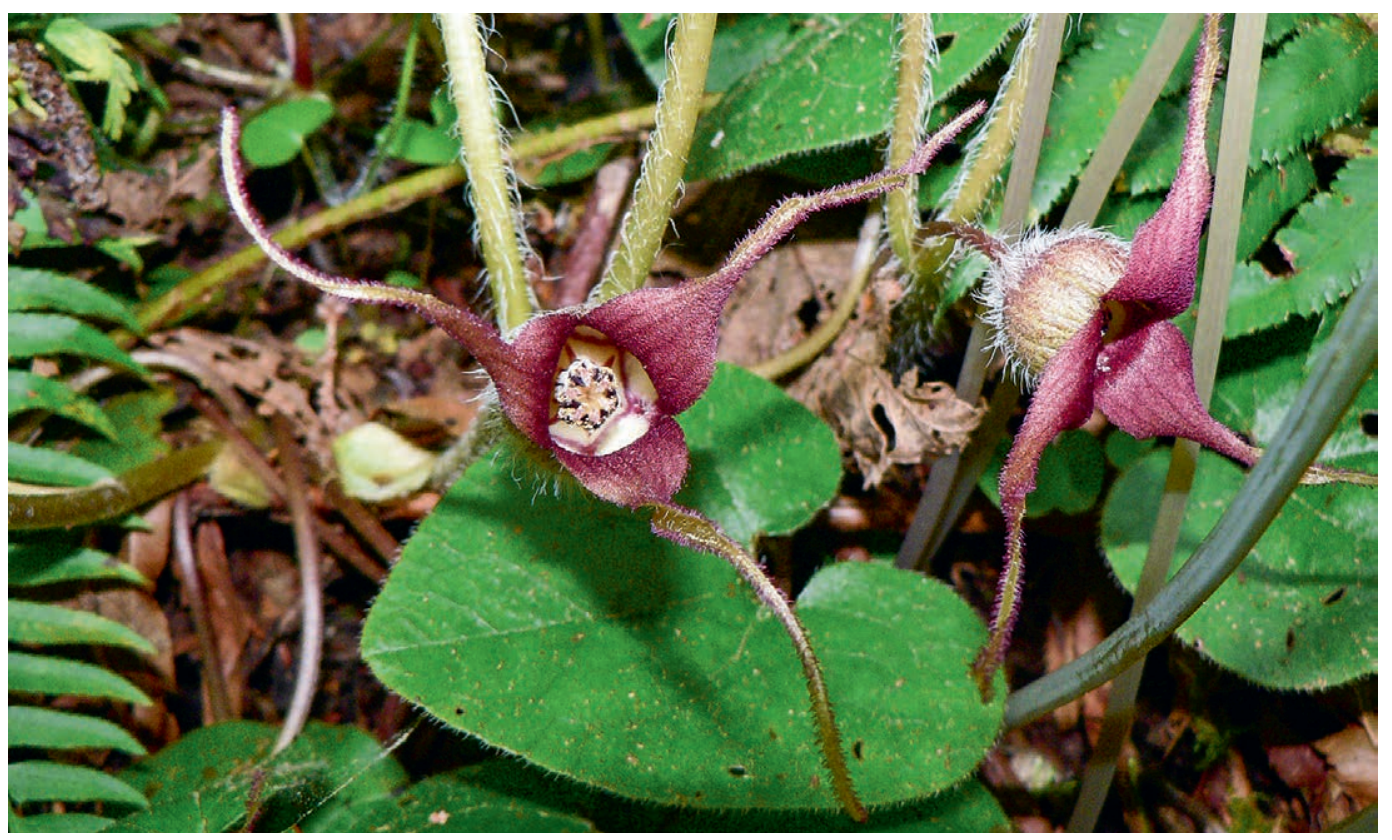

Abb. 1: Asarum caudatum (Aristolochiaceae) war eine der ersten Pflanzen, die STEFan Vogel als Pilzmückenblume erkannte und für die er eine detaillierte blütenökologisch-morphologische Analyse vorlegte. Zunächst studierte er Pflanzen, die im Botanischen Garten der Universität Mainz kultiviert wurden und konnte den Bestäubungsvorgang genau dokumentieren. (Foto: W. SiEgmund, https://en.wikipedia.org/wiki/Asarum_caudatum\#/media/File:Asarum_caudatum_10993.JPG; CC BY 2.5) 
gruppe. Fossil sind sie bereits aus dem Jura bekannt und in der Kreidezeit hatten sie bereits eine beachtliche Diversität entwickelt. Sehr bekannt sind die Pilzmücken als Einschlüsse im Bernstein.

Wie der Name besagt, ist das Leben vieler, wenn auch nicht aller, Arten mit dem Vorhandensein von Pilzen, genauer gesagt ihren Fruchtkörpern, verknüpft. Die Weibchen legen ihre Eier zwischen die Lamellen oder in die Poren von meist weichfleischigen Hutpilzen, also dort, wo es besonders feucht ist. Den daraus sich entwickelnden Maden dient das Fleisch der Fruchtkörper als Nahrung. Jeder Schwammerlsucher ist schon mit Pilzmücken in Berührung gekommen: das von Fraßgängen durchzogene madige Fleisch der Fruchtkörper geht auf die Anwesenheit von Pilzmückenlarven zurück.

Durch die Koppelung der Pilzmücken an (meist Boden-)Pilze ist der Lebensraum der Pilzmücken vorgegeben. Es ist der Waldboden mit Bodenkräutern, Moosen und verrottendem Laub. Die Luftfeuchtigkeit ist in diesem Mikrohabitat sehr hoch, die Lichtintensität gering. Pilzmücken haben kaum einen Austrocknungsschutz und sind daher nicht in der Lage, ihr angestammtes Habitat zu verlassen. Sie können zwar fliegen, tun dies aber selten.

Wenn sich in der Evolution Blüten an die Bestäubung durch Pilzmücken angepasst haben, dann müssen diese in dem gleichem Habitat vorkommen. Sie sind Bodenbewohner des Waldes, wo es schattig und luftfeucht ist. Ihre Blüten liegen oft direkt dem Boden auf oder sind halb vergraben. In tropischen Gefilden ist das Angebot an geeigneten Habitaten etwas größer. Dort kann man Pilzmücken und Pilzmückenblumen auch in der Kronenregion finden, insbesondere im kühlen, immerfeuchten und dauernd von Wolken durchwehten Bergwald.

Man muss sich im Klaren darüber sein, dass Pilzmückenblumen eigentlich Täuschorganismen und Parasiten sind: anthropomorph gesprochen, werden die Pilzmücken von den Pilzmückenblumen „in trügerischer Absicht“ angelockt und

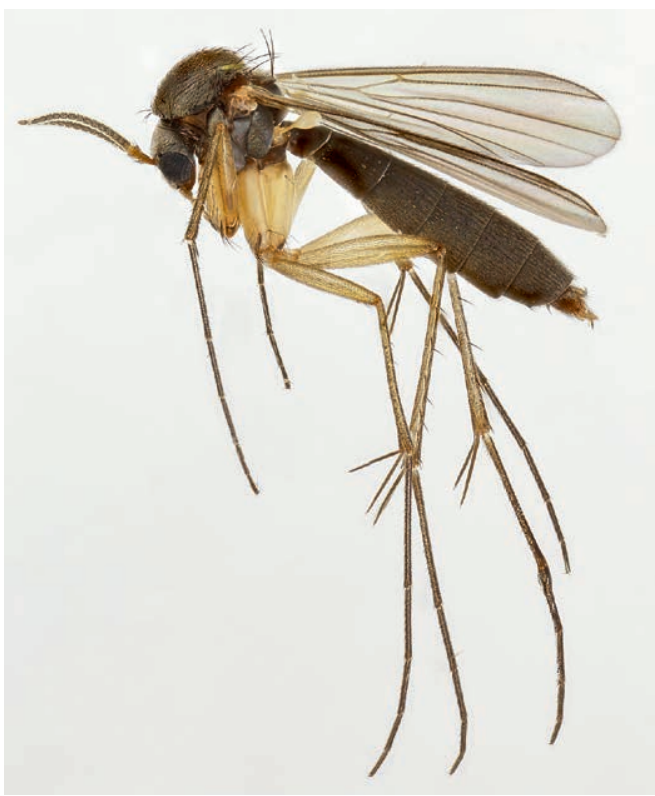

Abb. 2: Exechia spinuligera (Mycetophilidae), eine der zahllosen Arten von Pilzmücken. (Foto: J. Graham, https:// en.wikipedia.org/wiki/Mycetophilidae; CC BY 2.0)

zur Eiablage verleitet, wobei bei diesem Akt die Bestäubung vollzogen wird. Die sich aus den Eiern entwickelnden Maden finden keine Nahrung und sind dem Tode geweiht. Bei Arisaema dreht sich die Todesspirale sogar noch weiter: hier findet nicht nur der Nachwuchs, sondern auch die Blütenbesucher selbst den Tod, wenn sie nämlich an einen weiblichen Blütenstand geraten (s. u.).

Bei der Orchideengattung Dracula, die Vogel als Pilzmückenblumen bezeichnete, stellte sich mittlerweile heraus, dass sie z. T. auch von Taufliegen (Drosophiliden) bestäubt werden (ENDARA et al. 2010). Diese kleinen Fliegen tummeln sich gerne auf weißen Pilzfruchtkörpern. Auf den Dracula-Blüten zeigten die Männchen Balz- und Territorialverhalten, es kam sogar vereinzelt zur Paarung. Es handelt sich also hier nicht um eine Brutplatz-Attrappe, sondern um eine Balzarena, einen Rendezvousplatz.

\section{Anlockung der Pilzmüicken}

Von den echten Pilzfruchtkörpern werden die Pilzmücken sicher in erster Linie durch den charakteristischen Geruch, den jeder von frischen oder 
getrockneten Pilzen kennt, angelockt. Mit dem Thema, ob und wie die Pilzmückenblumen ihre Bestäuber olfaktorisch anziehen, hat sich VoGEL selbst nicht näher beschäftigt, aber als zukünftiges Forschungsgebiet empfohlen: „Eine Zukunftsaufgabe bleibt es ferner, die nicht wahrnehmbaren und die riechbaren Lockdüfte gaschromatographisch zu erfassen, genauer auf der Blüte zu lokalisieren und sie chemisch zu definieren" (Vogel 1978b: 395).

Leider wurden bisher nur sehr wenige Duftstoffanalysen von Pilzmückenblumen durchgeführt. Zu den Orchideen existieren bisher nur Publikationen von Kaiser (2006) zum Blütenduft von Dracula chestertonii, von Policha et al. (2016) zu Dracula lafleurii und von Lehnebach \& Zeller (2015) zu dem von Corybas-Arten. KaIser fand bei $D$. chesteronii eine Kombination von Oct-1-en-3-ol, Oct-1-en-3-one und Octan3-one, die perfekt an einen Pilzduft erinnern. Auch Polichia et al. (2016) fanden diese Substanzen mit noch weiteren wenig charakteristischen Duftstoffen. Nur sehr wenige der neuweltlichen Dracula-Arten weisen Pilzduft auf (D. chestertonii, D. chimaera, D. lafleurii, D. vampira). Die meisten Arten haben für die menschliche Nase keinen wahrnehmbaren Duft. Bei den altweltlichen Corybas-Arten konnte von LeHneBACH \& Zeller über 90 verschiedene Duftstoffe aus verschiedensten Substanzklassen (Monoterpene, Sesquiterpene, Diterpene, Fettsäurederivate, Steroide, Benzolderivate) nachgewiesen werden, wobei das gefundene 3-Octanol auch als Duftstoff von Pilzen bekannt ist. Hier muss noch erklärend hinzugefügt werden, dass Pilze nicht deshalb duften, um irgendwelche Organismen anzulocken, sondern dass es sich hier um olfaktorisch aktive Sekundärmetaboliten handelt.

Die von Vogel \& Martens (2000) publizierten Duftanalysen sind, wie die Autoren explizit ausdrücken, mit Vorsicht zu bewerten und als vorläufig anzusehen (s. o.). Die meisten Arisaema-Arten weisen einen sehr schwachen Duft auf, sind deshalb schwierig zu besammeln. Auch von R. KAISER zusammen mit dem Zweitautor (G. G.) durchgeführte Versuche, Düfte von verschiedenen
Arisaema-Arten zu sammeln, schlugen wegen der geringen Konzentration des jeweiligen Blütenduftes fehl. Nur Arisaema candidissimum konnte analysiert werden, aber die Duftanalysen weisen in eine völlig andere Richtung. Die rosa gefärbten mit breiten weißen Streifen versehenen Spathen gehören vermutlich in ein völlig anderes Bestäubungssyndrom. Der Duft ist sehr angenehm, er erinnert an Veilchen. In der Tat konnten hier $\beta$-Ionon und Dihydro- $\beta$-Ionon nachgewiesen werden zusammen mit einer höheren Konzentration an (E)-Nerolidol. Zusätzlich zum Duft müssen aber auch visuelle Merkmale eine gewisse Rolle spielen, sonst hätten die Pilzmückenblumen keine typisch „fungosen Strukturen“ entwickelt. Dazu zählt z. B. die Ausbildung von Längsstreifen (Arisarum vulgare, Arisaema) oder sogar dreidimensionale Nachahmung von Lamellen oder Waben (ostasiatische Arten von Asarum, Dracula-Arten sowie Arisaema griffithi). Wie das Beispiel von Asarum caudatum zeigt, ist eine visuelle Ähnlichkeit mit Pilzfruchtkörpern keine unbedingte Voraussetzung, Pilzmücken anzulocken. Allerdings ist die zunehmende Entwicklung von gestaltlichen Ähnlichkeiten mit Pilzfruchtkörpern - und das parallel in mehreren Verwandtschaftskreisen - sicher kein reiner Zufall. Wir wissen allerdings noch sehr wenig darüber. Polichia et al. (2016) machten Anlockungsexperimente mit Dracula-Blütenattrappen, die im 3-D-Druck erstellt und mit dem entsprechenden Duft versehen wurden. Sie wiesen nach, dass sowohl der Duft als auch die optischen Signale für die Bestäubung entscheidend sind. Die Blütenblätter steigern die Attraktivität der pilzartig geformten Lippe. Es ist bekannt, dass die Kombination von visuellen und olfaktorischen Signalen das Lernvermögen von Drosophila-Fliegen steigert.

\section{Aristolochiaceae: Asarum und Aristolochia}

Das erste Objekt, das Stefan Vogel eingehend studiert und als erstes eingehend beschrieben hat, ist Asarum caudatum aus der Familie der Osterluzeigewächse, Aristolochiaceae (Vogel (1978a). Es handelt sich um eine Art, die im westlichen Nordamerika beheimatet ist und im Botanischen Garten der Universität Mainz kultiviert wurde. Außerdem konnte er Pflanzen in Nordamerika unter natürlichen Bedingungen studieren. Im 
Gegensatz zu unserer heimischen Haselwurz (Asarum europaeum), einem obligaten Selbstbestäuber, konnte er bei $A$. caudatum Blütenbesuch bei $76 \%$ der Blüten der kultivierten Pflanzen und $35 \%$ der unter natürlichen Bedingungen in Amerika wachsenden Pflanzen beobachten und die Eiablage durch Pilzmücken (hauptsächlich Fungivora fungorum) dokumentieren. Im Schnitt konnte VoGEL vier Eier pro Blüte feststellen, wobei die Mücken pro Besuch in der Regel nur ein Ei ablegten. Die Ablage findet bevorzugt an der Wand der weißen Höhlungen statt, also dort, wo es besonders feucht ist.

Die Blüten weisen kaum eine äußere Ähnlichkeit mit Pilzen auf, tragen aber durch die braunrote Färbung und die lang geschwänzten Blütenblätter eindeutig Züge des Syndroms der Fliegenbestäubung (Myiophilie). Ebenso fehlt ein typischer Pilzgeruch. Dennoch erfolgt die Anlockung offenbar durch einen Duft, der aber für die menschliche Nase nicht wahrnehmbar ist.

In ausgeklügelten Experimenten konnte VoGEL nun zeigen, dass die (je zwei) weißen Höhlungen an der Basis der Blütenblätter eine hohe Luftfeuchtigkeit aufweisen und die bevorzugten Bereiche der Eiablage darstellen. Diese „feuchten Krypten" sind somit funktionell durchaus mit den feuchten Zwischenräumen der Lamellen von Pilzen zu vergleichen. Den Bestäubungsprozess muss man sich so vorstellen, dass die Pilzmücken beim Eindringen in die Blüte mit dem Buckel mit der Narbe in Berührung kommen und gegebenenfalls mitgebrachten Pollen an dieser abstreifen. Auch bei der Kopulation von Pilzmücken in der Blüte kann es zur Bestäubung kommen. All das passiert im weiblichen Stadium der Blüte, welches dem männlichen vorausgeht (Proterogynie). Im darauf folgenden männlichen Stadium der Blüten sind die Narben nicht mehr belegungsfähig und der freigesetzte Pollen wird auf dem Buckel des Insekts abgeladen.

Asarum caudatum ist nicht ausschließlich auf Fremdbestäubung durch Insekten angewiesen. Bleibt Insektenbesuch aus, kommt es zur Selbstbestäubung. Eine Studie an mehreren Populatio-

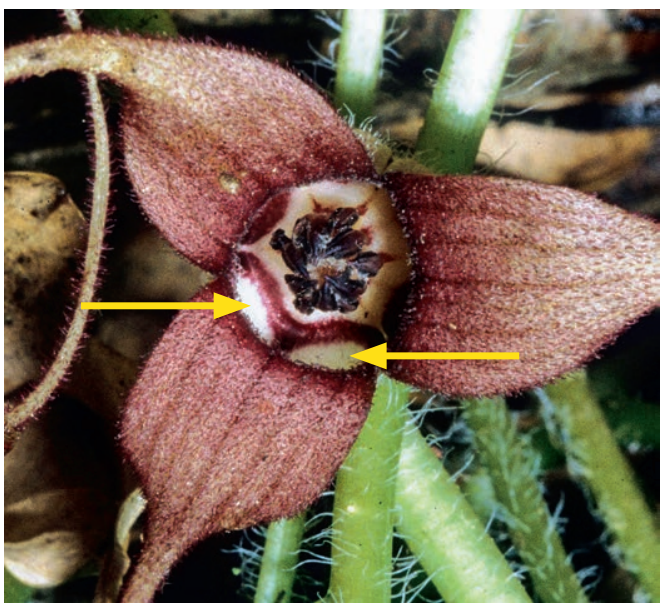

Abb. 3: Asarum caudatum (Aristolochiaceae). Blütenzentrum vergrößert; am unteren Blütenhüllblatt sind deutlich die beiden weißen Höhlungen („feuchte Krypten“) zu sehen, welche den bevorzugten Eiablageplatz darstellen, siehe Pfeile. (Foto: G. Gerlach)

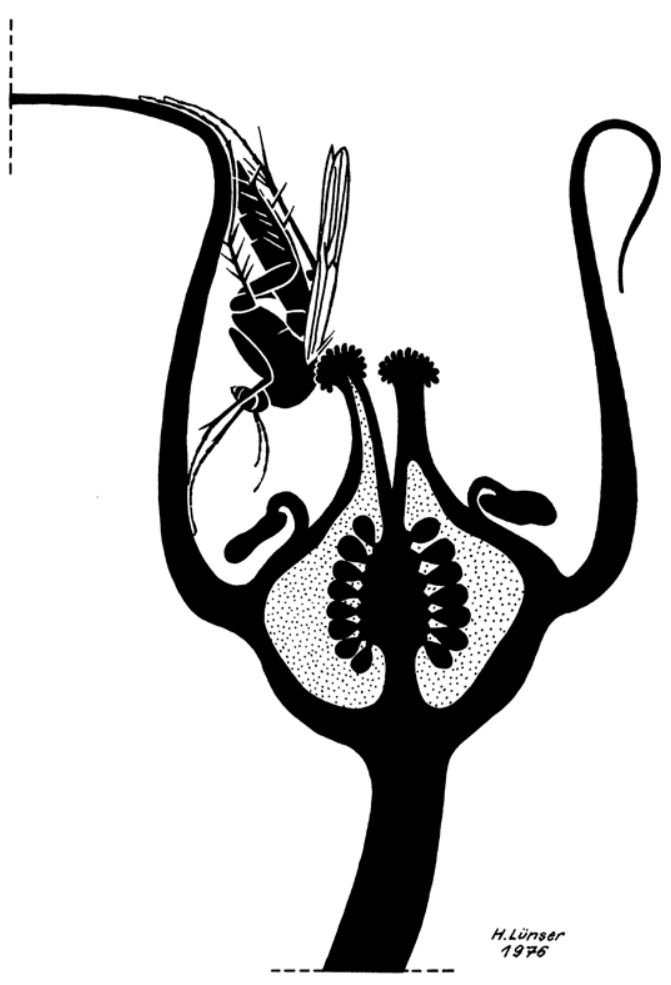

Abb. 4: Schema der Pollenabladung in einer Blüte von Asarum caudatum. Der aufgewölbte Thorax der Pilzmücke (Buckel) kommt in Kontakt mit der Narbe und eventuell mitgebrachter Pollen wird abgestreift. (Zeichnung H. LÜNSER, aus Vogel 1978a, Abb. 5a) 


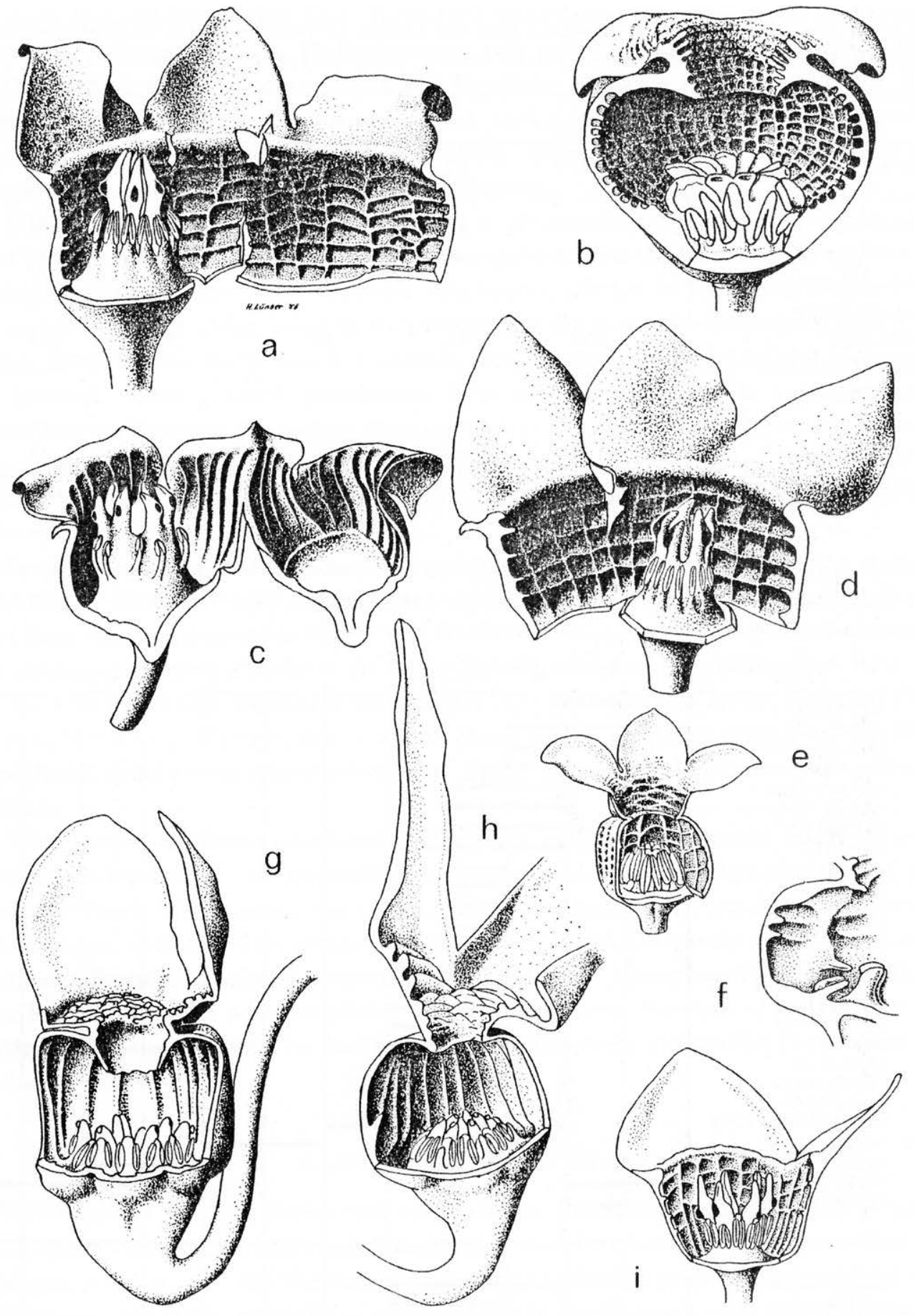

Abb. 5: Aufgeschnittene Blüten von ostasiatischen Asarum-Arten mit pilzähnlichen Strukturen; (a) A. megacalyx,

(b) A. asaroides, (c) A. sieboldii, (d) A. nipponicum, (e) A.parviflorum, (f) A. hexalobum, (g) A. costatum, (h) A. sakawanum, (i) A. takoi var. dilatatum. (Aus Vogel 1978a, Abb. 9, Zeichnungen H. Lünser) 
nen in Nord-Kalifornien ergab, dass dort Selbstbestäubung vorherrscht und Pilzmücken für die Bestäubung keine Rolle spielen (Lu 1982). Es scheint also regional große Unterschiede zu geben.

Wie gesagt, ist die Ähnlichkeit von Asarum caudatum mit einem Pilz nicht sehr groß. Betrachtet man aber ostasiatische Arten von Asarum, insbesondere jene, die der heute oft von Asarum abgetrennten Gattung Heterotropa angehören, so ist in jenem Bereich, in dem bei $A$. caudatum die sechs weißen Höhnlungen zu finden sind, ein Waben- (Asarum asaroides, A. megacalyx, A. nipponicaum u. a.) oder Lamellenmuster (A. costatum, A. sakawanum, $A$. sieboldii etc.) zu erkennen, das eindeutig pilzähnliche Züge trägt.

Die Hauptgattung der Aristolochiaceae ist Aristolochia, die mit etwa 500 Arten auf der ganzen Welt, besonders aber in den wärmeren Ländern, Subtropen und Tropen verbreitet ist. Ihre Blüten sind Kesselfallenblumen, die kleine Insekten (bes. Fliegen) anlocken, eine Zeit lang arretieren und nach Ausschüttung des Pollens die Tiere wieder freilassen. Diese tragen dann den Pollen zu anderen Blüten. Die meisten Aristolochia-Arten sind Kletterpflanzen und Lianen, nur vergleichsweise wenige Arten wie unsere heimische Aristolochia clematitis sind Bodenkräuter oder Sträucher (z.B. Aristolochia arborea). Die Blüten werden insbesondere bei den Kletterpflanzen im Luftraum postiert, also dort, wo Pilzmücken fehlen. Jene Arten, die sich auf die Bestäubung durch Pilzmücken spezialisiert haben, müssen daher ihre Fallenblüten in unmittelbarer Bodennähe aufstellen.

Es gibt mehrere Arten, die das tun und als Pilzmückenblumen oder zumindest als Vorstufen von solchen angesehen werden können. Das spektakulärste Beispiel ist ohne Zweifel Aristolochia arborea, ein Strauch, der in Mexico, Guatemala und El Salvador heimisch ist. Die Blüten werden, meist in größerer Zahl, an der Basis des Stammes gebildet, zuweilen, bei größeren, älteren Pflanzen aber auch weiter oben. Die Blütenhülle hat die Form einer weit offenen Kapuze. Der obere Teil ist braunrot, der untere Teil weiß gefärbt. Der

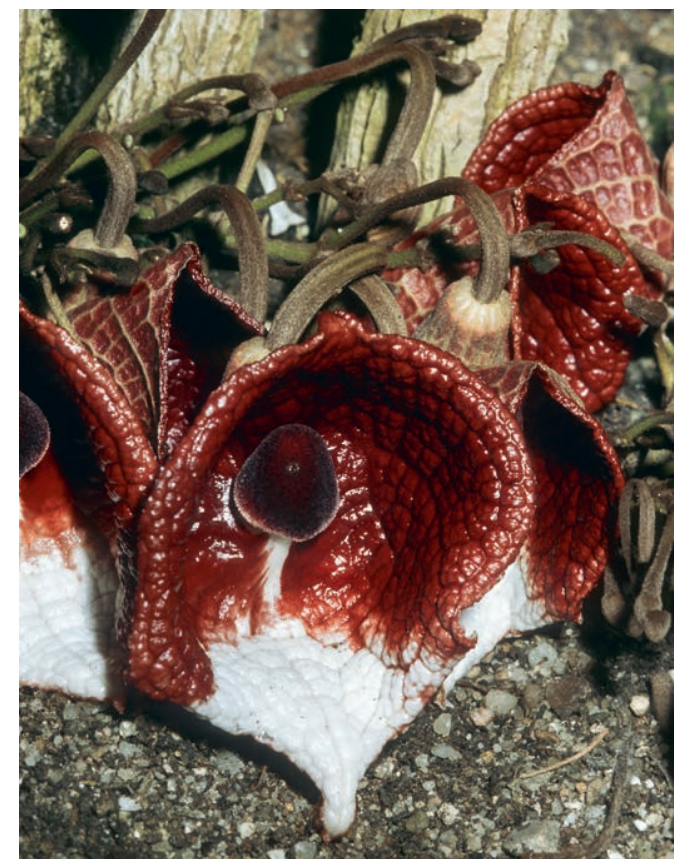

Abb. 6: Aristolochia arborea. Blüten mit Pilzattrappe vor dem (verdeckten) Eingang des Blütenkessels; der obere Pfeil weist auf den Hut, der untere auf den Stiel des Pseudo-Pilzes. Die Blüten werden vor allem an der Basis des Strauches gebildet. (Foto: G. Gerlach)

Kessel liegt auf der Hinterseite der Kapuze und in diesen müssen die Bestäuber hineinbugsiert werden. Das geschieht dadurch, dass am Kesseleingang (Schlund) eine perfekte Pilzattrappe postiert wird: ein „leibhaftiger, kleiner Hutpilz“, wie Vogel (1978a: 363) schreibt. Die Hutunterseite ist mit einem engmaschigen Lamellengewebe ausgestattet, in welches die Pilzmücken ihre Eier hineinlegen. Der Hut bzw. diese Gewebe auf der Hinterseite hat eine größere Ausdehnung, sodass die Tiere dorthin gelotst werden, wo sich genau darunter der Kesselschlund befindet. Eine kleine Unachtsamkeit, und die Mücke landet im Kessel. Sie kommt nach Abladen des eventuell mitgebrachten Pollens an der Narbe und Beladung mit neuem Pollen wieder frei. Sie ist "dumm genug“, in den Kessel der nächsten Blüte zu stürzen. Wenn auch Mücken im Kessel des Öfteren zugrunde gehen, ist der Tod der Tiere nicht vorprogrammiert. Im Gegensatz dazu überleben die Maden, die aus den abgelegten Eiern schlüpfen, mangels PilzfleischNahrung nicht. 

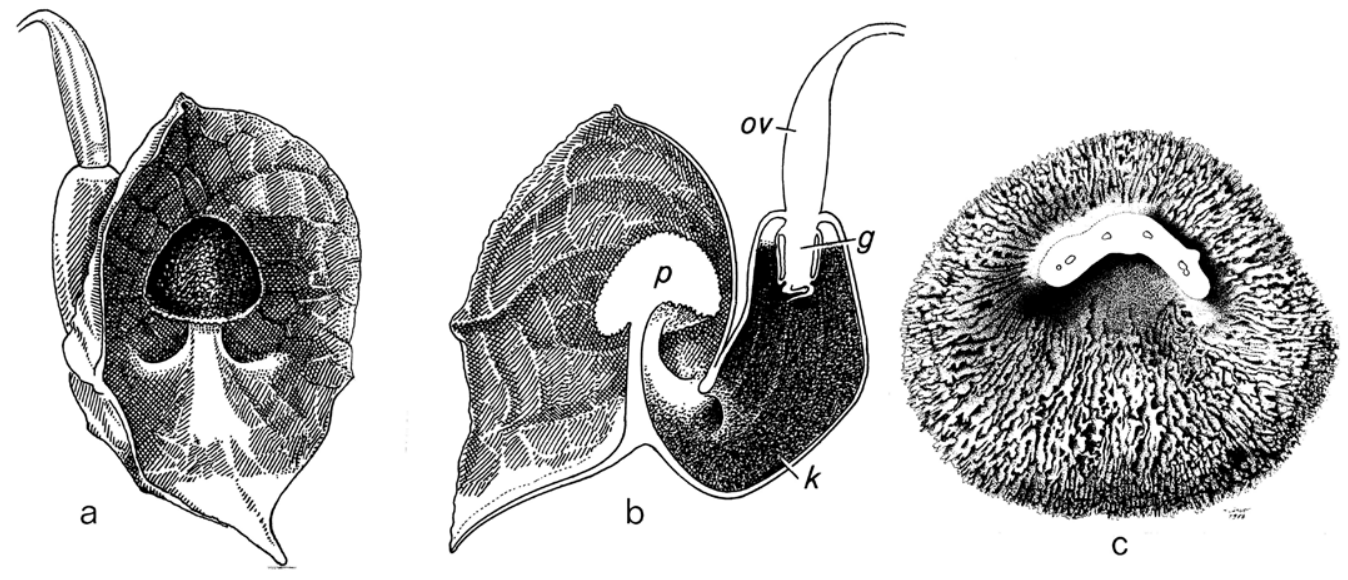

Abb. 7: Aristolochia arborea; (a) Blüte mit Pilzattrappe vor dem Kesseleingang; (b) Blüte median aufgeschnitten; (c) Unterseite des Pilzhutes; p - Pilzattrappe, g - Gynostemium, k - Kessel, ov - Fruchtknoten (Ovar). (Aus Vogel 1978a, Abb. 12).

\section{Araceae: Arisarum und Arisaema}

Bei den Aronstabgewächsen (Araceae) sind im Laufe der Evolution mehrfach Pilzmücken"blumen" entstanden. Dabei handelt sich allerdings immer um Blütenstände. Die Pilzähnlichkeit reicht von gering bis zu höchst erstaunlich. Beginnen wir mit ersteren: Im Mittelmeergebiet häufig ist Arisarum vulgare, eine Art, die im Verbreitungsgebiet von den Azoren und Kanaren bis in den Nahen Osten in mehrere Unterarten differenziert ist. Die Blütenstände sind etwa $10-15 \mathrm{~cm}$ hoch, die von einem Stiel emporgehobene, olivgrün-braunrot

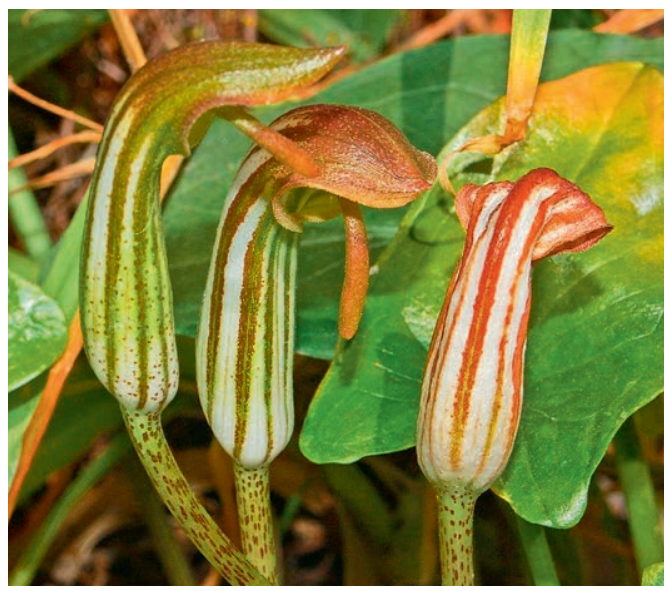

Abb. 8: Arisarum vulgare (Araceae): die Spathen der Blütenstände weisen longitudinale grüne bis bräunliche Streifen auf weißem Grund auf, die entfernt an die Lamellen eines Pilzes erinnern. (Foto: НестоNichus, https://en.commons.wikipedia.org/ File: Araceae-Arisarum vulgare-1.jpg) und durchscheinend weiß längsgestreifte Spatha bildet eine Röhre, deren Spitze sich haubenförmig über die Röhrenöffnung wölbt. Aus der Öffnung ragt der im oberen Teil schwach keulig verdickte und nach unten gebogene sterile Teil der Blütenstandsachse, der sog. Appendix, daher auch der deutsche Name „Krummstab“ für die Pflanze. Die Deutung, dass die haubenförmige Spathaspitze und die Streifung der Röhre etwas mit der Nachahmung eines Pilzes bzw. von Pilzlamellen zu tun hat, wird manchem sicher etwas weit hergeholt erscheinen.

Die zweite Art, A. simorrhinum, wird von manchen Botanikern nur als eine Unterart von A. vulgare betrachtet, die Hauptverbreitung liegt in der westlichen Hälfte Spaniens. Sie ist im Prinzip ähnlich, unterscheidet sich aber durch einen kürzeren Stiel, sodass der Blütenstand stärker in Bodennähe rückt. Der aufrechte Spadix zeigt eine ausgeprägt kopfige Verdickung des Appendix, sodass die Röhre fast verschlossen wird. Damit wird dem Funktionsprinzip einer Kesselfalle besser Genüge getan. Die Deutung als Pilzimitation wird aber noch nicht jeden überzeugen.

Doch bei der dritten Art der Gattung, A. proboscideum, kann kein Zweifel mehr bestehen. Die Pilzähnlichkeit ist schon dem italienischen Botaniker Giovanni Archangeli aufgefallen. doch erst Stefan Vogel konnte die Morphologie und 

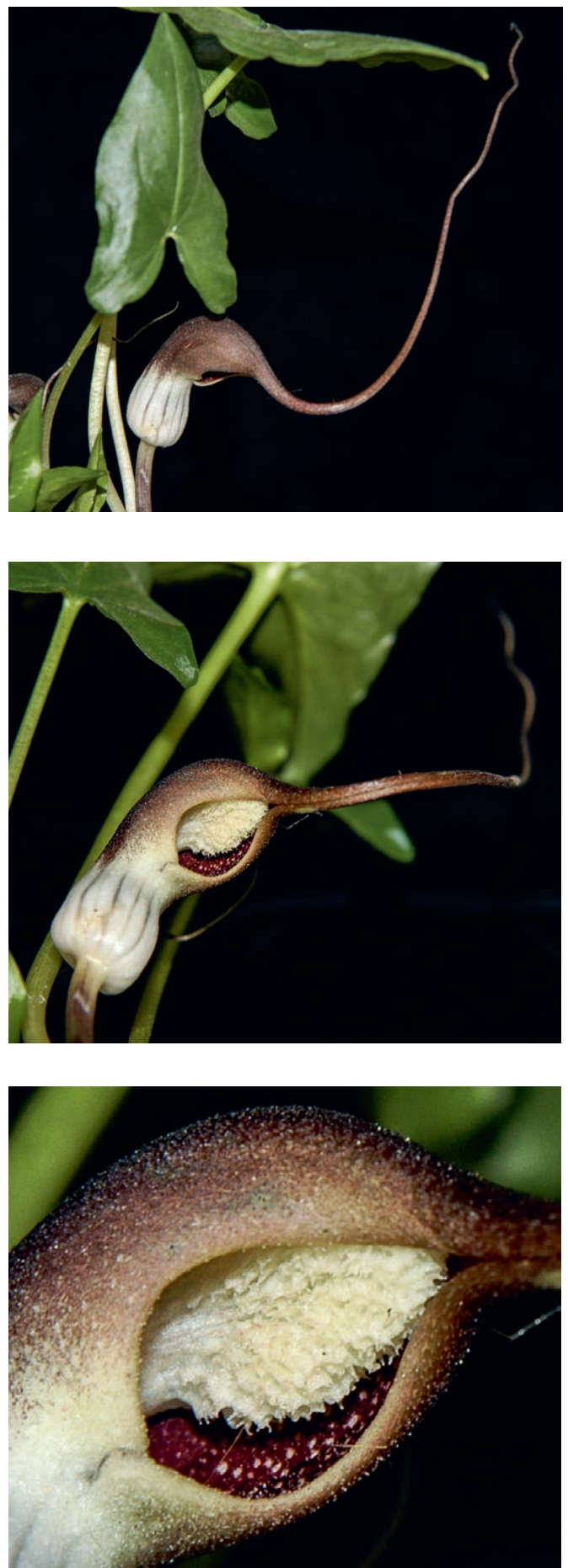

Abb. 9: Arisarum proboscideum. Oben: Pflanze mit Blütenstand in Seitenansicht. Mitte: Blütenstand von schräg unten. Unten: Ausschnitt aus dem Bild Mitte: fungoser Appendix mit lamellig-schwammiger Oberfläche. (Fotos: A. WeBER)
Blütenökologie dieser Art im Detail dokumentieren und in einen größeren Zusammenhang stellen (Archangeli 1891).

Diese Art ist in Mittel- und Süditalien sowie in Südwest-Spanien heimisch und kommt in submontanen, schattigen Laubwäldern zwischen 600 und 1000 Metern Seehöhe vor. Die Spatha ist im unteren Teil (Kessel) weißlich, im oberen Teil rotbraun gefärbt, die Spitze ist lang ausgezogen ist und zeigt steil aufwärts. Nicht von ungefähr wird die Pflanze in ihrem Heimatgebiet als Mauspflanze bezeichnet.

Im Gegensatz zu den beiden vorigen Arten ist die Spatha jedoch nicht geschlossen, sondern es bleibt an der dem Boden zugewendeten Seite ein Spalt offen. Der Spalt gibt den Blick frei auf einen großen, weißlichen Körper: den stark vergrößerten Kopf des Appendix, dessen Oberfläche eine lamellig-schwammige Struktur aufweist, unzweifelhaft eine Imitation eines Pilzes.

Die zweite Gattung der Araceen, die im Zusammenhang mit Pilzmückenbestäubung behandelt werden muss, ist Arisaema. Hier handelt es sich um eine große Gattung, die mit etwa 250 Arten in Asien (Himalaya, China, Japan, SOAsien), Afrika und mit wenigen Arten in Nordund Mittelamerika verbreitet ist.

Im Gegensatz zu Arisarum und anderen Araceen sind die Pflanzen diözisch, es gibt also männ-
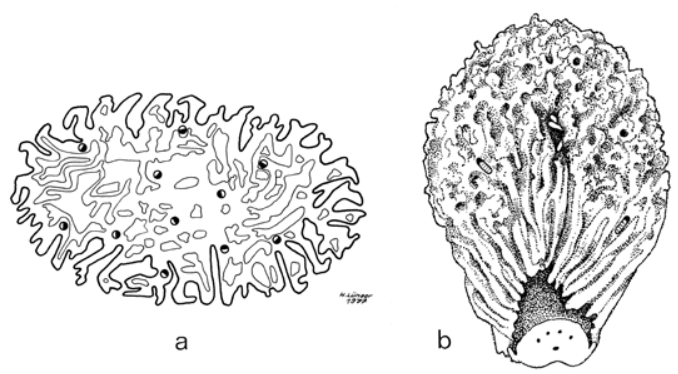

Abb. 10: Arisarum proboscideum; (a) Querschnitt durch den fungosen Kopf des Appendix mit Gewebelakunen und Gefäßbündeln; (b) Appendix von unten, in der Mitte drei Eier, auf den Lamellen zwei junge Larven und oben Bohrgänge von Fungivora fungorum. (Aus Vogel 1978b, Abb. 15) 


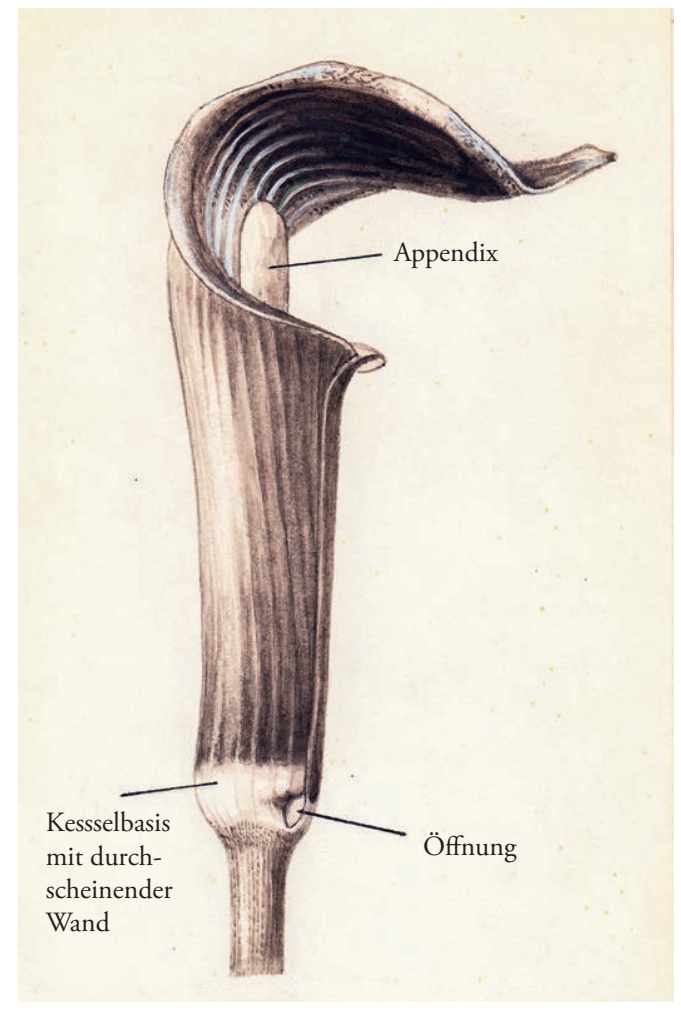

Abb. 11: Arisaema sp. (Araceae), Blütenstand einer männlichen Pflanze; nach der Pollenausschüttung hat sich am Kesselgrund eine Öffnung gebildet. (S. VoGel, unpubl. Handzeichnung)
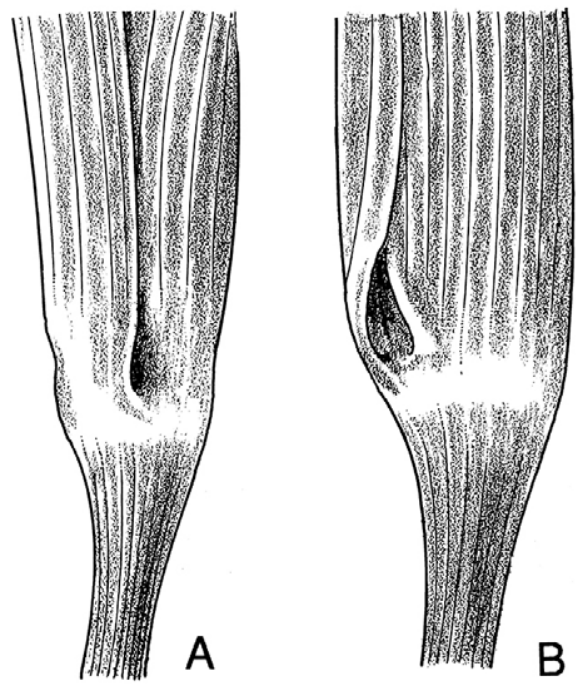

liche und weibliche Pflanzen. Bei Arisarum ist das Prinzip der Kesselfallen nur „halbherzig“ verwirklicht. Die Insekten fallen wohl in einen Kessel, werden aber nicht in Gefangenschaft gehalten, sondern können jederzeit den Kessel wieder verlassen. Dagegen ist das Kesselfallenprinzip bei Arisaema perfekt realisiert. Die weiblichen Fallen stellen hier tödliche Gefängnisse dar, aus denen es kein Entkommen gibt. Sobald die Staubblätter gereift sind und der Pollen ausgeschüttet wurde, sammelt er sich sich am Boden des Kessels, wo die Mücken herumkrabbeln. In den männlichen Fallen geben die Ränder der Spatha an der Basis des Kessels eine Öffnung frei, durch welche die Insekten den Kessel verlassen können. Geraten die Insekten jedoch in eine weibliche Falle, bleibt der Kessel geschlossen. Der mitgebrachte Pollen sichert der Pflanze die Bestäubung und Befruchtung und somit den Nachwuchs in Form der Samen. Die Tiere können nicht mehr entweichen und sind dem Tod durch Verhungern preisgegeben.

Besonders erwähnenswert sind jene Arten, bei denen der Appendix extrem verlängert, nach unten gebogen und $z$. T. ausgefasert ist. Im natürlichen Habitat liegen diese Strukturen dem Boden auf, sind in der Laubstreu vergraben und bilden Leitstrukturen für die Pilzmücken, die somit zu

Abb. 12: Arisaema triphyllum; (A-C) Männlicher Blütenstand, Öffnung an der Kesselbasis (in C Kessel aufgeschnitten, Spadix

entfernt); (D) Weiblicher Blütenstand; der Kessel öffnet sich nicht. (Aus Vogel \& Martens 2000, Fig. 4)
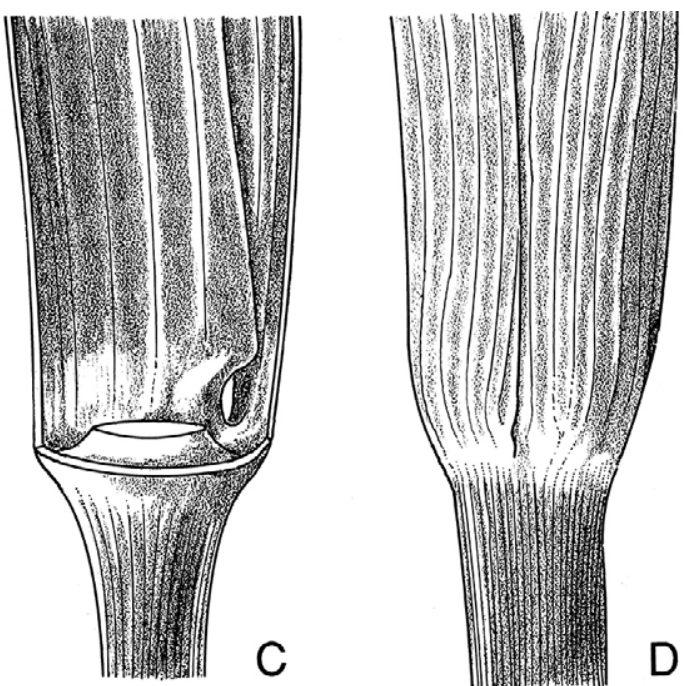
Fuß zum Kessel gelangen können. Ein eindrucksvolles Beispiel liefert $A$. griffithii. Die Spathen der Blütenstände sind helmförmig gekrümmt, überdachen den Eingang zur Kesselfalle und tragen ein auffälliges Streifen- und Netzmuster. Die Blütenstände sehen dadurch wie große, überreife Pilzfruchtkörper aus. Die Appendices der Blütenkolben sind extrem zu einem Flagellum verlängert, ihre Spitzen liegen in der Bodenstreu vergraben. Sie fungieren als Anlockungs- (Duft!) und Leitstrukturen für die in der Waldstreu lebenden Pilzmücken.

Eine exemplarische Studie über die Bestäubung von neun Arisaema-Arten des Himalaya-Gebiets haben später Vogel \& Martens (2000) vorgelegt. Als Hauptbestäuber konnten Pilzmücken aus den Familien Mycetophilidae und Sciaridae (insgesamt 16 Gattungen und 47 identifizierte Arten, darunter eine neue Gattung und 22 neue Arten) festgestellt werden. Die Spezifität ist somit nicht sehr hoch und als Blütenbesucher/Bestäuber fungieren sowohl Männchen als auch Weibchen.

\section{Orchidaceae: Corybas, Cypripedium und Dracula}

Die stets einblütigen, faszinierenden Winzlinge der Gattung Corybas (zwischen 100 und 150 Arten) wachsen wie die von ihnen nachgeahmten Pilze terrestrisch in dunklen feuchten Wäldern. Sie weisen ein weites Verbreitungsgebiet vom Himalaya bis S-China und Polynesien bis Australien auf. Wegen ihrer oft lang geschwänzten Blüten werden sie auch als Spinnen-Orchideen bezeichnet. Da die Bestäubung unter dem kapuzenförmig über die restlichen Blütenorgane gestülpten dorsalen Sepalum erfolgt, liegen keine Schilderungen des eigentlichen Bestäubungsvorgangs vor. Es wird aber häufiger über Corybas-Pollinien tragende Pilzmücken (Arte der Gattung Mycetophilia, Mycetophilidae) berichtet und diese wurden auch in der Umgebung der blühenden Pflanzen nachgewiesen. Die Pollinien werden den Pilzmücken auf den deutlich buckelförmigen Thorax geklebt. Die Anlockung erfolgt über den Blütenduft, der sehr verschieden sein kann. Häufig können Eier und Larven der Pilzmücken auf den Lippen von Corybas spp. gefunden werden. Somit ist der Beweis erbracht,

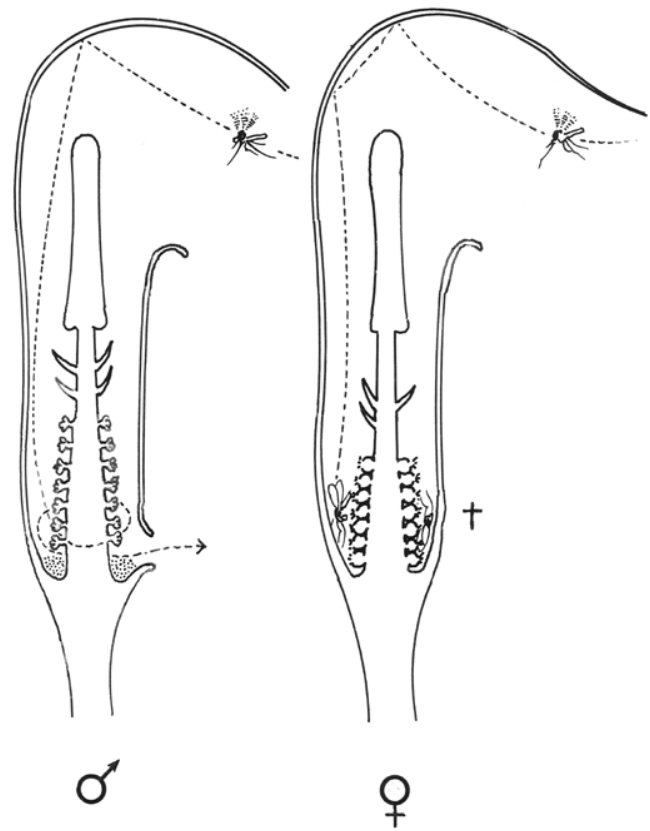

Abb. 13: Schema der Pollenübertragung bei Arisaema (Araceae). Links: Männlicher Blütenstand: nachdem die Pilzmücken in den Kessel gestürzt und mit Pollen eingestäubt sind, können sie den Kessel durch eine sich bildende Öffnung wieder verlassen. Rechts: Weiblicher Blütenstand: der Kessel bleibt geschlossen, die gefangenen Insekten sind dem Tod durch Verhungern preisgegeben. (Aus Vogel \& Martens 2000, Abb. 6)

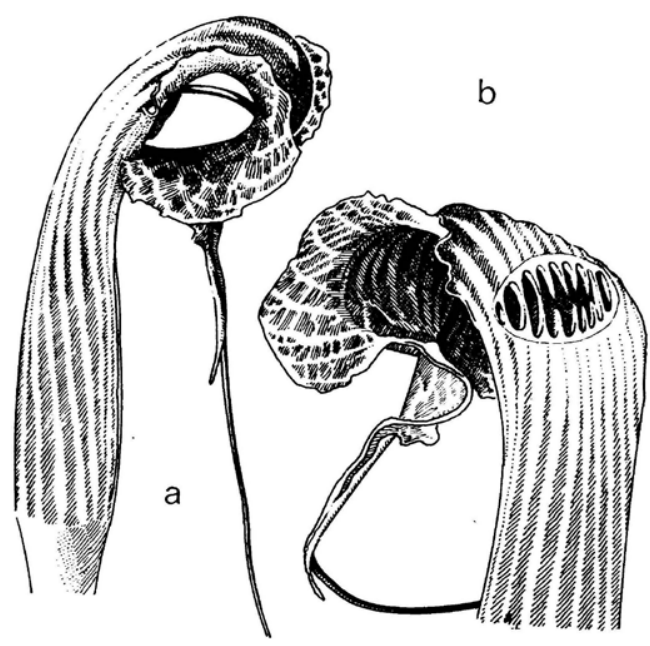

Abb. 14: Arisaema utile, Spatha seitlich (a) und dorsal (b); in (b) der Spatharücken tangential angeschnitten, sodass die dreidimensionale Struktur der Rippen sichtbar wird; der Appendix ist fadenförmig und hängt aus der Spatha heraus. (Aus Vogel 1978b, Abb. 17) 


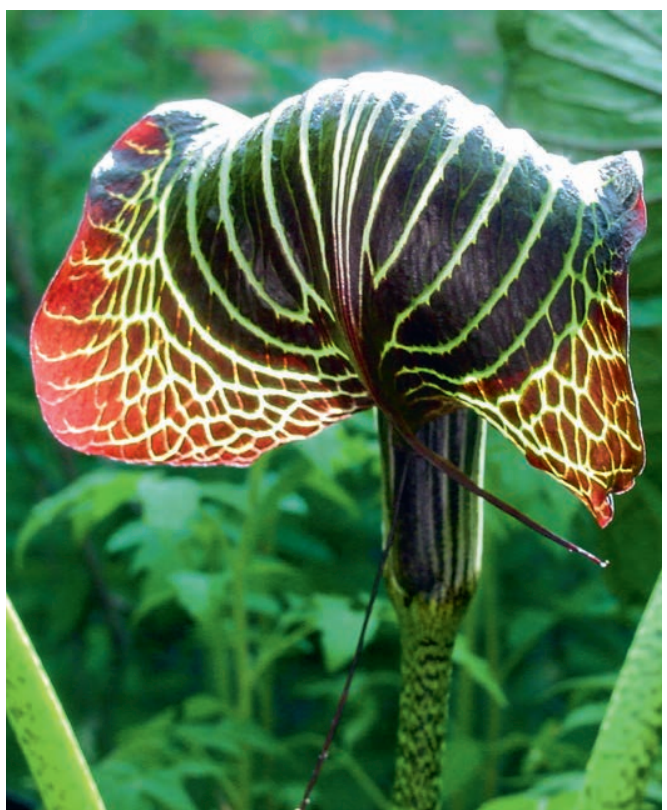

Abb. 15a: Arisaema griffithii. Blütenstand von vorne gesehen; der Eingang in den Kessel wird durch den umgeschlagenen Oberteil der Spatha verdeckt (Foto: R. Hammond)

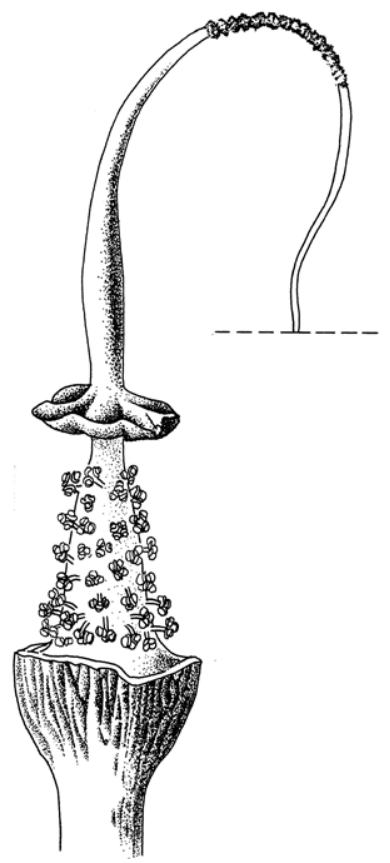

Abb. 15 c: Freipräparierter Spadix; der sterile Appendix ist an der Basis zu einem hut(pilz)ähnlichen Gebilde verbreitert, der distale Teil hängt über und die lang ausgezogene Spitze (nicht dargestellt) ist in der Laubstreu vergraben. (Aus Vogel 1978b)

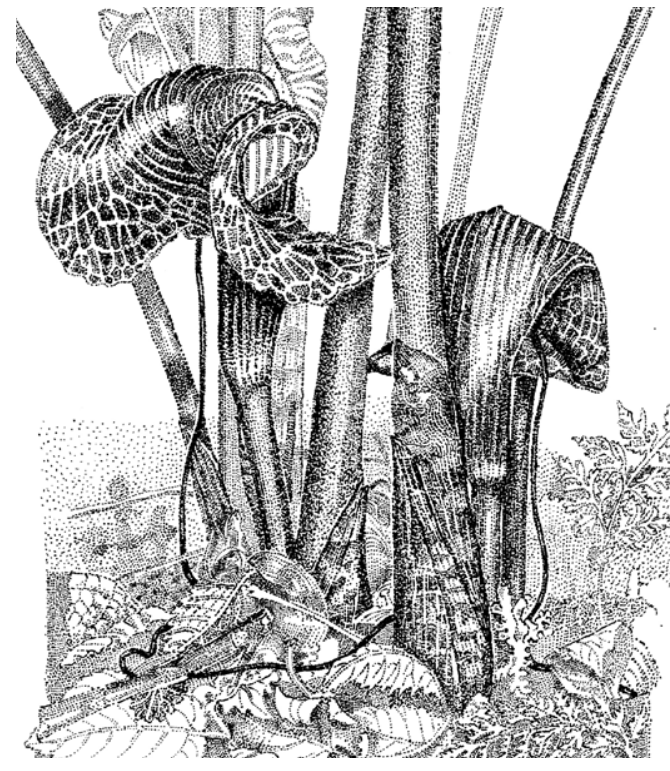

Abb. 15b: Zwei Blütenstände von Arisaema griffithii; die langen und dünnen Appendices hängen nach unten und ihre Spitzen sind in der Waldstreu vergraben, Näheres siehe Text. (Aus Vogel \& Martens 2000)

dass es sich um Brutplatzmimeten handelt. Die beiden hier abgebildeten Corybas-Arten stammen aus Neuseeland und sind erst seit wenigen Jahren bekannt (LehnebaCh 2016, Lehnebach et al. 2016). Näheres über pilzmückenbestäubte Orchideen Neuseelands ist in den unten genannten Internetlinks finden.

Die von Vogel erwähnten Pterostylis-Arten (ca. 200 spp., vor allem Australien und Neuseeland) werden ebenfalls von Mycetophilidae, aber auch von Keroplatidae bestäubt. Hier handelt es sich zwar um Pilzmückenblumen, aber mehr um Sexualtäuschblumen, denn es wurden nur männliche Tiere von den Blüten angelockt und als pollinientragend nachgewiesen (Reiter et al. 2019).

Die Gattung Dracula wurde erst 1978 von dem amerikanischen Orchidologen Carlyle A. Luer beschrieben. Neben neubeschriebenen Arten beinhaltet sie viele Arten, die ursprünglich der Gattung Masdevallia zugeordnet wurden. Der Name ist nach Luer (1978) die Verkleinerungsform vom Lateinischen draco, Drache, bedeutet also kleiner Drache. Aber natürlich assoziiert wohl 

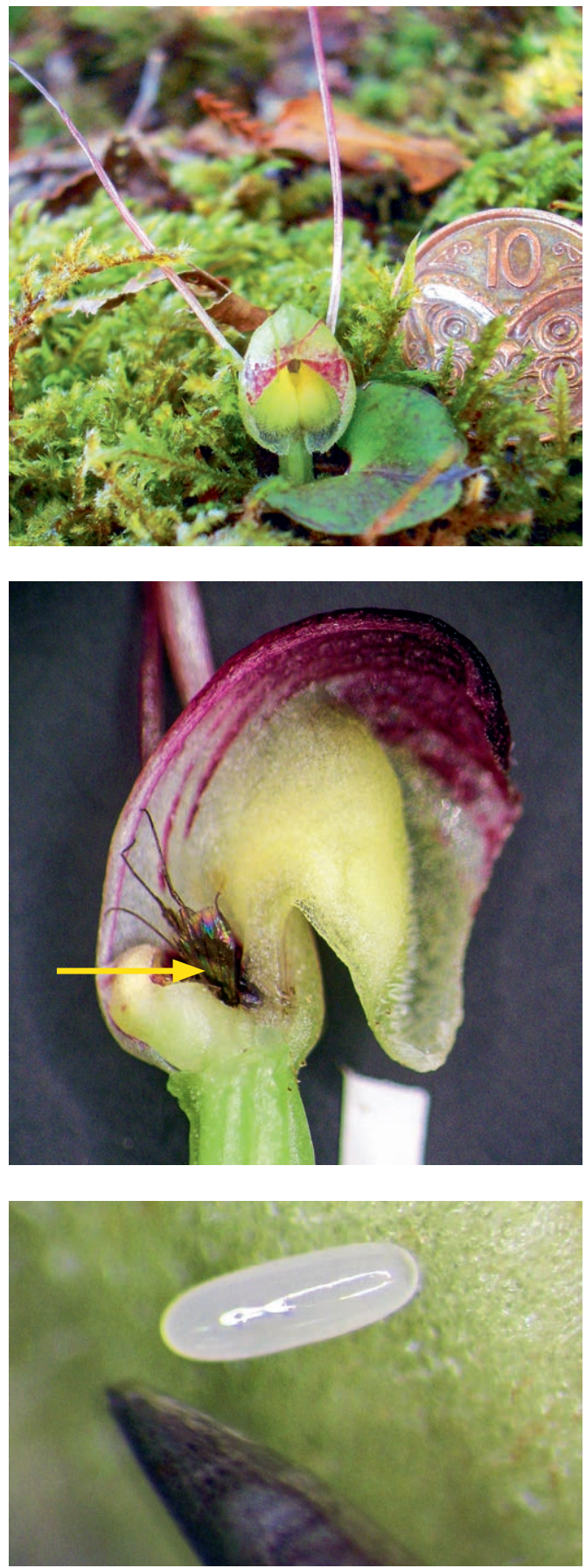

Abb. 16: Corybas walliae (Orchidaceae). Oben: Pflänzchen mit zweischwänziger Blüte; die 10-Cent-Münze misst im Durchmesser etwa $20 \mathrm{~mm}$. Mitte: Aufgeschnittene Blüte mit eingeklemmter Pilzmücke (Mycetophila spec.; Pfeil). Unten: In der Blüte abgelegtes Ei (Größenvergleich: Spitze einer Präpariernadel). (Fotos: C. Lehnebach)
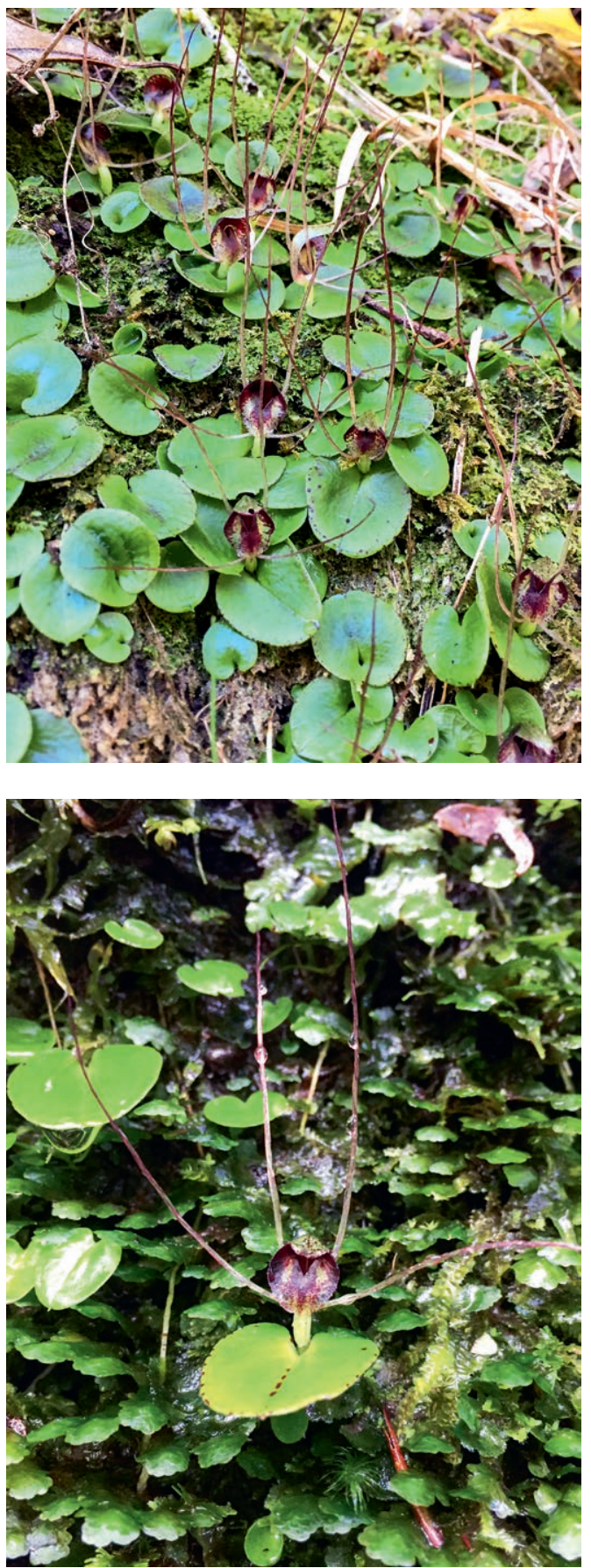

Abb. 17: Corybas hatchii (Orchidaceae). Oben: Bestand von blühenden Pflanzen auf einer feuchten, moosbewachsenen Wand. Unten: Pflanze mit vier zu langen und dünnen Schwänzen ausgezogenen seitlichen Sepalen und Petalen. (Fotos: C. Lehnebach, Neuseeland, Nordinsel, Remutaka Range) 


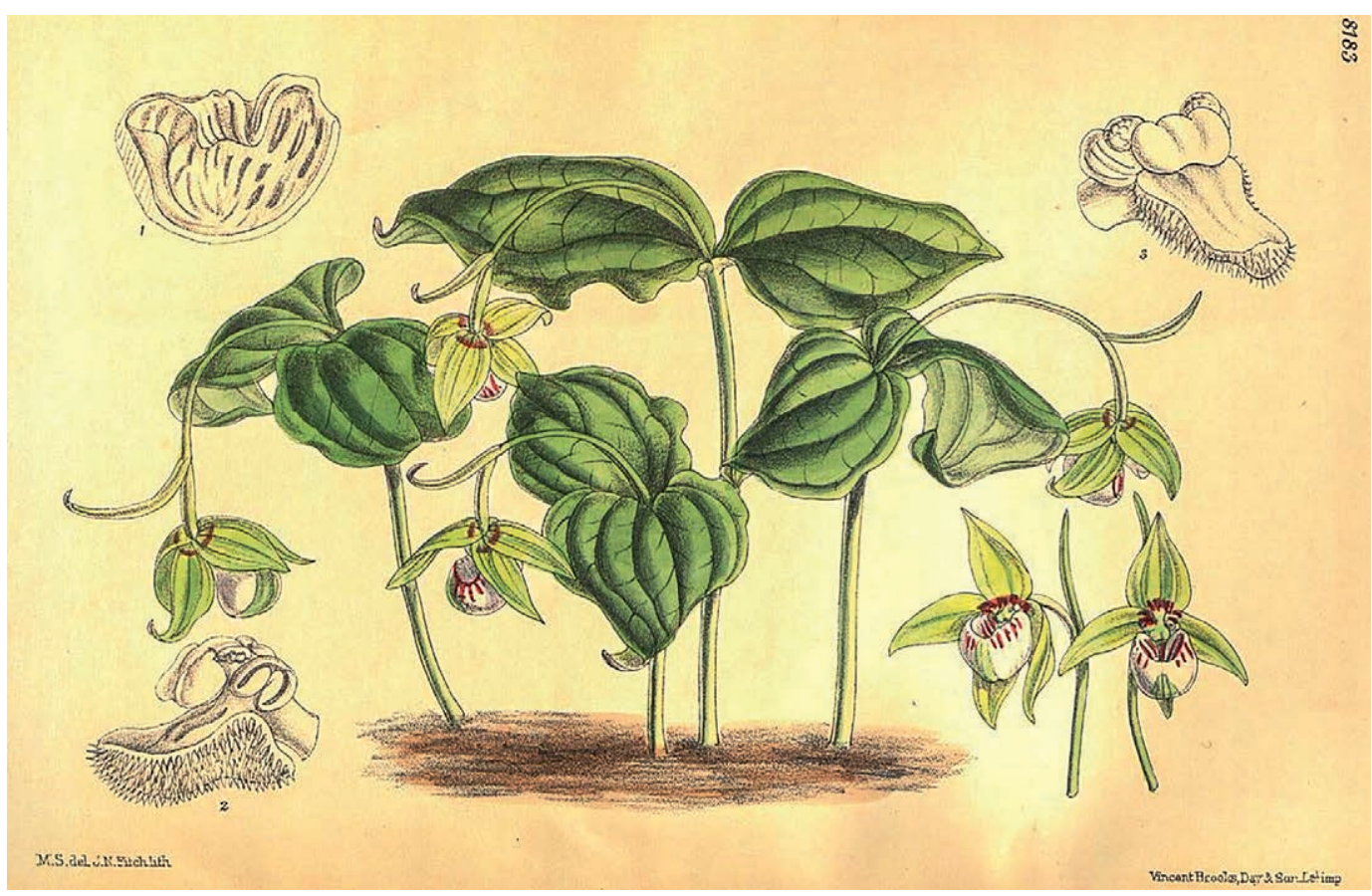

Abb. 18: Cypripedium debile (Orchidaceae). Die winzigen Pflänzchen dieser japanischen Zwergorchidee bilden eine einzige Blüte in hängender Position, dadurch ist die Öffnung des Labellums nach schräg-unten orientiert und die am Boden lebenden Fliegen können die Lippenöffnung leicht erreichen und in das Blütenzentrum vordringen. (Aus CurTis's Bot. Mag. 134, pl. $8183,1908)$

jeder mit dem Namen die Roman- und Filmfigur des Grafen Dracula, die der irische Schriftsteller Bram Stoker 1897 in Anlehnung an den historischen Herrscher VLAD III. mit dem Beinamen DrĂCUlEA (1431-1476/77) geschaffen hat und

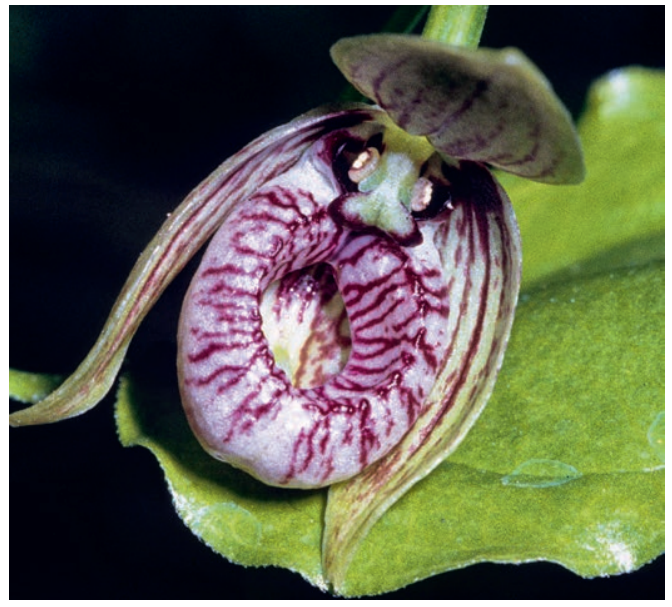

Abb. 19: Cypripedium debile (Orchidaceae); Blüte, Lippe von oben gesehen. (Foto: G. Gerlach) der im Roman in Transsilvanien sein Unwesen als Vampir getrieben hat. Tatsächlich weisen die Blüten vieler Arten eine frappante Ähnlichkeit mit einem Gesicht, oder besser gesagt, einer menschlichen, tierischen oder teuflischen Fratze auf: die Augen werden durch die dunklen Petalen, die Nase durch die Säule und der bezahnte Mund durch das Labellum mit den „Pilzlamellen“ gebildet, die drei äußeren Tepalen mit ihren meist langen Schwänzen ähneln Fledermausflügeln. Tafeln mit Blüten zahlreicher Dracula-Arten sind z. B. in den am Ende genannten Internetseiten zu finden.

Die Dracula-Arten sind allesamt Epiphyten in den sehr feuchten Nebelwäldern der Gebirgsstufen Süd- und Mittelamerikas. Sie wachsen im Schatten auf den unteren Ästen und Zweigen von Bäumen, die mit einer feuchten Moos- oder Humusschicht bedeckt sind. Die Blüten liegen zuweilen mit dem „Gesicht nach unten“ der Unterlage auf (Abb. 20). Die wenig flugfreudigen Pilzmücken können 


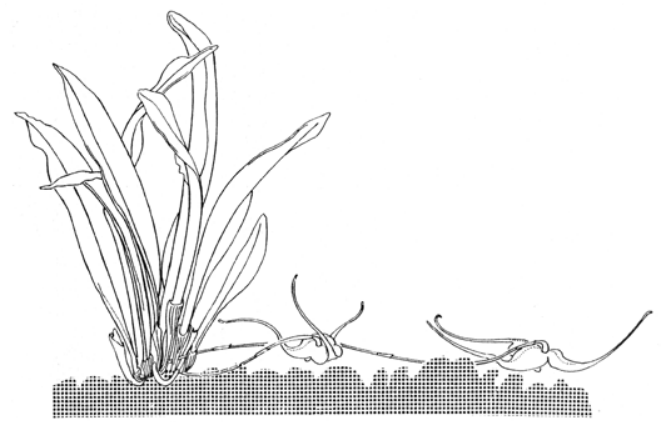

Abb. 20: Dracula erythrochaete, ein Epiphyt des zentralamerikanischen Bergregenwaldes, zeigt die typische Wuchsform der Dracula- und vieler Masdevallia-Arten: die Blüten liegen mit dem „Gesicht nach unten“ der Decke epiphytischer

Moose auf. (Aus Vogel 1978b, als Masdevallia erythrochaete)

daher ganz leicht zu den pilzähnlichen Labellen der Blüten gelangen und dort ihre Eier ablegen.

Der Gattungsname, die kuriosen Blüten und phantasievoll-schaurige Artnamen wie Dracula diabola (Teufels-Dracula), D. gorgona (Gorgonenhaupt-D.), D. nosferatu (Nosferatu-D.), D. vampira
(Vampir-D., Abb. 21), D. veneficus (Vergifter-D.) etc. haben dazu beigetragen, Dracula zu einer der bekanntesten tropischen Orchideengattungen zu machen, mit der tragischen Konsequenz, dass viele Arten am Naturstandort ausgerottet wurden. Wie C. A. Luer in einem Gespräch mit dem Erstautor sagte, bereue er zutiefst, für diese Orchideengruppe den Namen Dracula vergeben zu haben. Danach hätten sich nämlich Scharen von Orchideen,freunden" und botanischen Trophäenjägern auf den Weg gemacht, diese Orchideen an ihren meist eng begrenzten Naturstandorten rücksichtslos zu sammeln und damit zu dezimieren oder ganz auszurotten.

In einer Orchideengärtnerei bei Medellín in Kolumbien wurden zahlreiche Dracula-Arten gemeinsam unter naturnahen Bedingungen kultiviert. Erstaunlicherweise fanden sich dort immer wieder „neue“ Arten, die Wissenschaftler fanden es rätselhaft, wie diese den Weg in die Sammlung fanden. Schließlich wurde erkannt, dass es Jungpflanzen waren, die aus den Samen der Kapseln entstammten. Nun war es rasch klar, dass sich

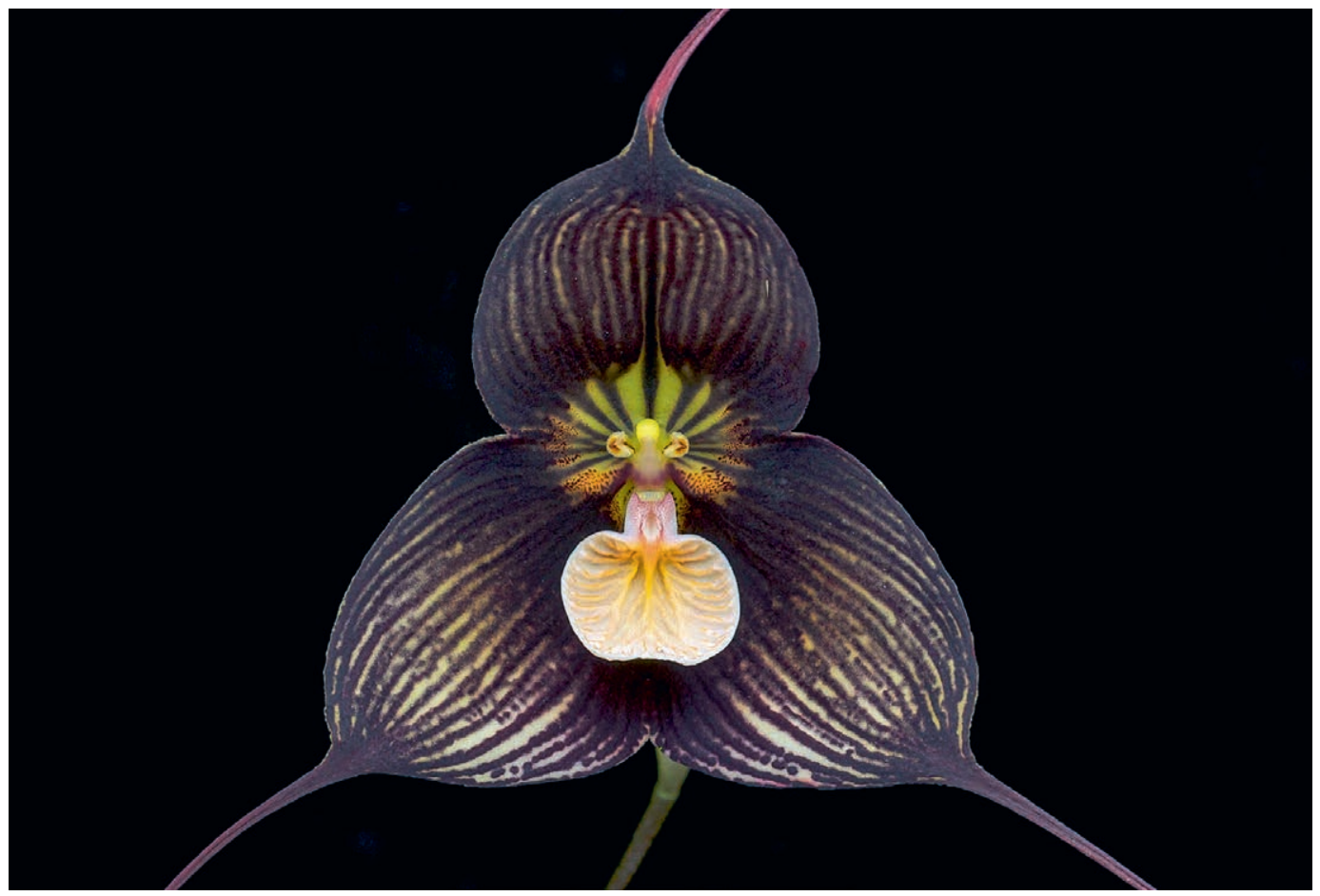

Abb. 21: Dracula vampira. (Foto: E. Hunt, https://commons.wikimedia.org/wiki/File:Dracula_vampira_3.jpg; CC BY-SA 3.0.) 

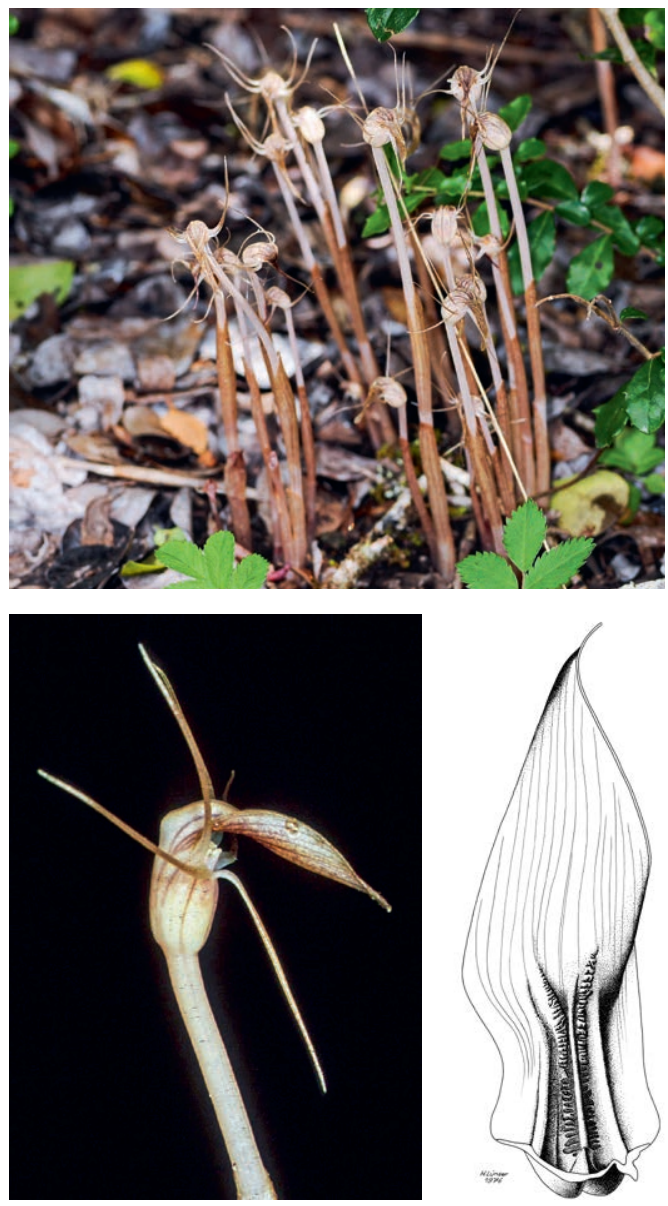

Abb. 22: Arachnitis uniflora (Corsiaceae). Oben: Gruppe von Pflanzen (Foto: A. SÉrsic; Chile, Araucanía-Llancalil, 333 m). Mitte: Blüte in Seitenansicht (Foto: A. SÉrsIC; Argentinien, Prov. Neuquén, Dep. Los Lagos, Villa La Angostura, Laguna Verde, 813 m). Rechts: Helmblatt von unten gesehen. (Aus Vogel 1978b).

Hybriden gebildet hatten. Die Dracula-Arten hatten wohl keine hohe Bestäuberspezifität, so dass die dort in der Gärtnerei vorkommenden Pilzmücken spontan Hybriden erzeugten und die Wissenschaftler an der Nase herumführten. In der Natur gibt es diese Hybriden nicht, denn die verschiedenen Arten wachsen geografisch getrennt, die Artintegrität wird durch geografische Barrieren gewährleistet.

Endara et al. (2010) beschreiben detailliert den Bestäubungsvorgang. Taufliegen der Gattung
Zygothrica (Drosophilidae) landen auf den Sepalen und laufen zielgerichtet zur Lamellen tragenden Lippe. Ständig die Lippen-Oberfläche beleckend, bewegen sie sich von dort zur Basis der Lippe in eine Kammer, gebildet von Säule, Lippe und den Petalen. Beim weiteren Vordringen in diese Kammer drücken sie mit den Hinterbeinen die bewegliche Lippe von der Säule weg und ihr Rücken wird von den einwärts gekrümmten Rändern des klauenförmigen Rostellums (ein steriler Teil des mittleren Narbenlappens der Orchideen) eingeklemmt. Nun sind alle weiteren Versuche, sich zu befreien, erfolglos. Nachdem das Tier immer wieder Pausen in seinen Befreiungsversuchen einlegt, kehrt die Lippe in die ursprüngliche Position zurück und drückt den Bestäuber leicht gegen die Säule. Schließlich wird das Scutellum der Fliege mit einer klebrigen Flüssigkeit benetzt und die Caudikel (Stielchen) der Pollinien dort festgeklebt. Endlich wird die Fliege vom Rostellum freigegeben. Der ganze Vorgang dauert fast eine Stunde. So ist es erklärlich, dass man nicht selten eingeklemmte Fliegen auf Dracula-Blüten findet.

\section{Corsiaceae: Arachnitis}

Die Corsiaceae sind eine kleine, nur drei Gattungen (Corsia, Corsiopsis, und Arachnitis) umfassende Familie. Ihre ca. 25 Arten sind alle chlorophylllos und mycoheterotroph, repräsentieren also nichtgrüne Pflanzen, die auf Pilzen parasitieren (wie wir das z. B. von der heimischen Nestwurz, Neottia nidus-avis, kennen). Möglicherweise sind alle drei Gattungen pilzmückenbestäubt, Vogel (1978b) behandelt allerdings nur die einzige Art von Arachnitis, nämlich $A$. uniflora, aus den kühleren Teilen Südamerika. Auch diese wird nur fragmentarisch behandelt, da er, bedingt durch den Zeitpunkt des Auffindens im Südwinter, nur abgeblühte Exemplare untersuchen und keinen Blütenbesuch oder Eiablage feststellen konnte.

Die oberirdischen Teile der Pflanze umfassen nur einen Stängel mit einer terminalen Blüte, die nur wenige Zentimeter aus der Laubstreu herausragt. Durch die ausgezogenen Spitzen der lateralen Blütenblätter wirkt die Blüte spinnenartig. Das obere, äußere Blütenblatt (Sepalum) ist größer als die anderen und wölbt sich helmförmig über den 
Blüteneingang. Allerdings verschließt es diesen nicht vollständig, sodass kleine Blütenbesucher wie höchstwahrscheinlich Pilzmücken in die Blüte gelangen können. Das Helmblatt trägt beiderseits der Mittelrippe zwei Längsfurchen, die durch kleine Querstege leitersprossenartig unterteilt sind. Somit ergibt sich ein Wabenmuster, das entfernt an die oben geschilderten ostasiatischen AsarumArten erinnert.

Die morphologischen Merkmale und das Habitat von Arachnitis uniflora sprechen klar für die Bestäubung durch kleine Fliegen und man wird wohl nicht in der Annahme Vogels fehlgehen, dass es sich um Pilzmücken handelt. Das gleiche gilt vielleicht auch für die anderen Corsiaceen, konkrete Studien oder Beobachtungen fehlen allerdings völlig.

\section{Schlussbemerkungen}

Mit dem vorliegenden Beitrag geht unsere mehrteilige Serie in der Zeitschrift „Der Palmengarten " über die großen wissenschaftlichen Leistungen und Entdeckungen von Stefan Vogel zu Ende. Vogel hat im Laufe seines Arbeitslebens zahlreiche Entdeckungen gemacht. Dabei handelt es sich keineswegs um kleine und vernachlässigbare Beiträge. Sie bauen allerdings größtenteils auf schon bekannten Fakten auf und sind von VogeL weiterentwickelt oder monographisch behandelt worden. Nehmen wir als Beispiel die Kesselfallenblumen. Das sind Blüten oder Blütenstände, die, anthropomorph gesprochen, in „trügerischer Weise" Insekten anlocken, sie am Rand einer kesselförmigen Struktur zum Absturz bringen, dort eine Zeitlang gefangen halten, und dann mit Pollen beladen wieder freilassen. Obwohl in den Grundzügen schon seit langem bekannt, zieht sich die morphologische und funktionelle Analyse von Kesselfallenblumen wie ein roter Faden durch das gesamte Lebenswerk Vogels und ist in mannigfacher Weise mit den großen Entdeckungen verknüpft. Erste Beispiele hat Vogel schon in seinem Südafrika-Buch (Vogel 1954) behandelt, spezielle Beiträge (Ceropegia: Vogel 1961) und Überblicksarbeiten (Vogel 1965) folgten in den 1960er-Jahren. Über die tödlichen Kesselfallen von Arisaema (auf der Basis der Arbeiten von Vogel \& Mer-
TENS 2000) wurde in diesem Beitrag berichtet. Wie ein Plenarvortrag anlässlich der 12. Jahrestagung der Gesellschaft für Tropenökologie (VoGEL 1999) und spätere Vorträge (die nicht durch Publikationen dokumentiert sind) zeigen, plante VoGEL offenbar ein großangelegtes, zusammenfassendes Werk über Kesselfallenblumen. Dazu ist es leider nicht mehr gekommen.

In unserer Serie nicht erwähnt wurde u. a. die wichtige Kategorie der Pollen- und Pollentäuschblumen. Auch sie hätten wohl einen eigenen Beitrag verdient. VoGeL hat exemplarisch gezeigt, wie aus klassischen Pollenblumen, also Blumen, die an Stelle von Nektar Pollen als Beköstigungsmittel für die Bestäuber (ausschließlich Bienen) bereitstellen, durch Auslagerung von optischen Signalen schrittweise Pollentäuschblumen entstanden sind (Vogel 1978c).

Das klassische Pendant zu den Pollenblumen sind die Nektarblumen. In diesem Zusammenhang ist die vierteilige Serie über „bemerkenswerte Nektarien“ (Vogel 1997, 1998a-c) bzw. Einzelarbeiten über die Nektarien und den Nektar der Malvaceae (Vogel 2000), und einiger Combretaceae, Cucurbitaceae und Bignoniaceae zu erwähnen (Lopes et al. 2002, Vogel 1981, Weber \& Vogel 1986). Auch Nektarblumen können durch bloßes Vortäuschen von Nektar (glitzernde Körperchen) zu Täuschblumen werden.

Pollen- und Nektartäuschblumen stellen Pollen- (oder Antheren-) bzw. Nektar(tröpfchen)attrappen zur Schau, um Insekten anzulocken. Die im vorliegenden Beitrag behandelten Pilzmückenblumen gehören hingegen nicht in diese Kategorie, sondern in die Gruppe der Brutsubstrat-Täuschblumen und funktionieren teilweise nach dem Prinzip der Kesselfalle (Aristolochia, Arisarum, Arisaema, Cypripedium). Den bisher besprochenen Täuschblumen hat Vogel neben Einzelarbeiten ein eigenes Büchlein gewidmet, in dem neben der Erklärung des Grundprinzpis (Sender und Empfänger von optischen, olfaktorischen und taktilen Signalen) zahlreiche Beispiele aus allen möglichen Verwandtschaftsgruppen der Blütenpflanzen zusammengestellt sind (Vogel 1993). 
Doch es gibt noch eine weitere Kategorie von Täuschblumen, die unter dem Titel „Anlockung durch Bewegung" figurieren kann. Es handelt sich um Blüten, die Flimmerkörper ausprägen: feine, am Ende kopfig verdickte Haare oder kleine Gewebeläppchen, die mit der Blüte gelenkig verbunden sind und beim leistesten Lufhauch in eine flirrendzitternde Bewegung geraten. Die Adressaten sind Fliegen, die die flimmenden Körperchen für Artgenossen halten. Einen ersten Überblick hat VoGEL schon 1954 gegeben, ihnen dann aber fast 50 Jahre später eine umfangreiche monographische Studie gewidmet (Vogel 2002).

Selbst die letzte Arbeit, die Vogel noch weitgehend fertigstellen konnte, deren Erscheinen er aber nicht mehr erlebt hat, ist eine großangelegte Studie. Sie behandelt die Bestäubung tropischer und subtropischer Korbblütler durch Wirbeltiere: Vögel, Fledermäuse und nicht-fliegende Säugetiere (Vogel 2015).

Wenn im Lebenswerk von STEFAn Vogel auch blütenökologische Arbeiten bei weitem dominieren, dürfen die Studien zu anderen botanischen Teilgebiete nicht unerwähnt bleiben. Als Beispiel sei etwa die dreiteilige Publikationsserie über die Lotosblume (Nelumbo nucifera, Vogel 2004a,b, Vogel \& HadACEK 2004), welche unter anderem das Durchlüftungssystem dieser Wasserpflanze zum Gegenstand hat (Vogel 2004a). Mit der Öko-Morphologie von Wüstenpflanzen befasst sich ein weiterer Artikel aus neuerer Zeit. Dabei geht es um die sog. "Curly-Whirlies“"von Südafrika, insbesondere dem Namaqualand (Vogel \& Müller-Doblies 2011). Das sind hauptsächlich monokotyle Pflanzen, die spiralig gewundene oder sonstwie aberrant geformte Blätter (Oberflächenvergrößerung!) haben, mit denen sie Feuchtigkeit aus der Luft (Nebel) kämmen. Die Feuchtigkeit kondensiert an den kuriosen Blättern und wird in Form von Wassertröpfchen in den Boden geleitet. Von dort wird das Wasser von den Wurzeln aufgenommen.

Alle diese genannten Werke und viele andere, die aus Raumgründen hier nicht angesprochen werden können, stellen beileibe keine kleinen wissenschaftlichen Leistungen dar. Das Lebens- werk VogeLs ist fast unerschöpflich und wird noch vielen Forschern und Forschergenerationen eine Grundlage und Anregung für weitergehende Studien bieten. Eine Liste von Vogels Publikationen findet sich in Weber \& Sontag 2006 (19502005) und Weber 2016 (2006-2015). Stefan Vogel hat uns am 5. November 2015 für immer verlassen. Einer der zahlreichen Nachrufe ist in Heft 79/2015 dieser Zeitschrift erschienen.

\section{Literatur}

Arcangeli, G. 1891: Sull'Arisarum proboscideum Savi. Nuov. Giorn. Bot. Ital. 23: 545-549.

Endara, L, Grimaldi, D. A. \& Roy, B. A. 2010: Lord of the flies: pollination of Dracula orchids. - Lankesteriana 10: $1-11$.

KaIser, R. 2006. Flowers and fungi use scents to mimic each other. - Science 311: 806-807.

Lehnebach, C. A. 2016: New combinations and a replacement name for three New Zealand spider orchids (Corybas). New Zealand Native Orchid J. 139: 4-5.

Lehnebach, C. \& Zeller, A. 2015. Dissecting the smell of deception; the chemistry behind the pollination system of $\mathrm{New}$ Zealand spider orchids. - Orchids: 65-70.

Lehnebach, C. A., Zeller, A. J., Frericks, J. \& Ritchie, P. 2016: Five new species of Corybas (Diurideae, Orchidaceae) endemic to New Zealand and phylogeny of the Nematoceras clade. - Phytotaxa 270: 1-24.

Lopes, A. V., Vogel, S. \& Machado, I. C. 2002: Secretory trichomes, a substitute floral nectar source in Lundia A.DC. (Bignoniaceae), a genus lacking a functional disc. - Ann. Bot. 90: $169-174$.

Lu, K. L. 1982: Pollination biology of Asarum caudatum (Aristolochiaceae) in northern California. - Syst. Bot. 7:150-157.

Luer, C. A. 1978: Dracula, a new genus in the Pleurothallidinae. - Selbyana 2: 190-198.

Policha, T., Davis, A., Barnadas, M., Dentinger, B.T.M, Raguso, R.A. \& Roy, B.A. 2016: Disentangling visual and olfactory signals in mushroom-mimicking Dracula orchids using realistic three-dimensional printed flowers - visual and olfactory signals. - New Phytol. 210: 1058-1071.

Reiter, N., Freestone, M., Brown, G. \& Peakall, R. 2019. Pollination by sexual deception of fungus gnats (Keroplatidae and Mycetophilidae) in two clades of Pterostylis (Orchidaceae). - Bot. J. Linn. Soc. 190: 101-116.

Vogel, S. 1954: Blütenbiologische Typen als Elemente der Sippengliederung, dargestellt anhand der Flora Südafrikas. Bot. Studien, Heft 1, 1-338. Jena: Fischer.

Vogel, S. 1961: Die Bestäubung der Kesselfallenblumen von Ceropegia. - Beitr. Biol. Pflanzen 36: 159-237. 
Vogel, S. 1965: Kesselfallenblumen. - Umschau Wiss. \& Technik 65: 12-17.

Vogel, S. 1978a: Pilzmückenblumen als Pilzmimeten. - Flora 167: 329-366.

Vogel, S. 1978b: Pilzmückenblumen als Pilzmimeten. Fortsetzung und Schluß. - Flora 167: 367-398.

Vogel, S. 1978c: Evolutionary shifts from reward to deception in pollen flowers. In: Richards, A. J. (ed.): The pollination of flowers by insects. - Linn. Soc. Sympos. Series 6: 89-96.

Vogel, S. 1981: Trichomatische Blütennektarien bei Cucurbitaceen. - Beitr. Biol. Pflanzen 55: 325-353.

Vogel, S. 1997: Remarkable nectaries: structure, ecology, organophyletic perspectives. I. Substitutive nectaries. - Flora 192: 305-333.

VogeL, S. 1998a: Remarkable nectaries: structure, ecology, organophyletic perspectives. II. Nectarioles. - Flora 193: 1-29.

VogeL, S. 1998b: Remarkable nectaries: structure, ecology, organophyletic perspectives. III. Nectar ducts. - Flora 193: 113-131

VogeL, S. 1998c: Remarkable nectaries: structure, ecology, organophyletic perspectives. IV. Miscellaneous cases. - Flora 193: $225-248$

Vogel, S. 1999: Die Funktionstypen der Kesselfallenblumen. - 12. Jahrestagung der Gesellschaft für Tropenökologie (gtö), 17-19 Feb. 1999, Ulm (D), Tagungsband (Abstracts), Plenarvortrag V-1.1, p. 20.

Vogel, S. 2000: The floral nectaries of Malvaceae sensu lato: a conspectus. - Kurtziana 28: 155-171.

Vogel, S. 2002: Flickering bodies: Floral attraction by movement. - Beitr. Biol. Pflanzen 72: 89-154.

Vogel, S. 2004a: Contributions to the functional anatomy and biology of Nelumbo nucifera (Nelumbonaceae). I. Pathways of air circulation. - Plant Syst. Evol. 249: 9-25.

Vogel, S. 2004b: Contributions to the functional anatomy and biology of Nelumbo nucifera (Nelumbonaceae). II. Unique emergent druses on the floral receptacle. - Plant Syst. Evol. 249: $27-35$.

VogeL, S. 2015. Vertebrate pollination in Compositae: floral syndromes and field observations. - Stapfia:reports 103: 5-26.

Vogel, S. \& Hadacek, F. 2004: Contributions to the functional anatomy and biology of Nelumbo nucifera (Nelumbonaceae). III. An ecological reappraisal of floral organs. - Plant Syst. Evol. 249: 173-189.

Vogel, S. \& Martens, J. 2000: A survey of the function of the lethal kettle traps of Arisaema (Araceae), with records of pollinating fungus gnats from Nepal. - Bot. J. Linn. Soc. (London) 133: 61-100.

Vogel, S. \& Müller-Doblies, U. 2011: Desert geophytes under dew and fog: the "curly-whirlies" of Namaqualand (South Africa). - Flora 206: 3-31.

Weber, A. 2016: In memorian Stefan Vogel (1925-2015). Flora 218: 92-93.
Weber, A. \& Sontag, S. 2006: Stefan Vogel 80 years - a life devoted to floral ecology. - Flora 201: 331-339.

Weber, A. \& Steinecke, H. 2017: Die großen wissenschaftlichen Leistungen von Stefan Vogel (1925-2015): Teil 3. Blütendüfte und ihre Bildung in den Osmophoren. - Palmengarten 81: 69-77.

Weber, A. \& Vogel, S. 1986: The pollination syndrome of Deplanchea tetraphylla (Bignoniaceae). - Plant Syst. Evol. 154: 237-250.

\section{Internetseiten}

https://www.sciencedirect.com/science/article/pii/S0367253 017311246 (Artikel Vogel 1978a; kostenpflichtig)

https://www.sciencedirect.com/science/article/pii/S0367253 017311301 (Artikel Vogel 1978b; kostenpflichtig)

http://www.online-keys.net/sciaroidea/add01/Vogel_\&_ Martens_2000_Pollination.pdf (Artikel Vogel \& MARTens 2000).

https://www.rnz.co.nz/national/programmes/ourchanging world/audio/201825731/when-orchids-smell-likemushrooms-a-tale-of-botanical-deceit (Pilzmückenbestäubte Orchideen Neuseelands)

http://4.bp.blogspot.com/-of3Gxby3_GU/UqhNirPVxAI/ AAAAAAAAA $1 \mathrm{k} / 0 \mathrm{vI}$ KKB $1 \mathrm{rNo} / \mathrm{s} 1600 /$ Monkey+face+ Orchid+species.jpg

https://image.dhgate.com/0x0/f2/albu/g4/M01/2B/18/rB VaEVnovmWAFCGSAAMqkVD2wak624.jpg,

http://morzsafarm.hu/wp-content/uploads/2017/09/ezekneka-viragoknak-tenyleg-arcuk-van-majomkepu-orcidea.jpg (Fotos verschiedener Dracula-Arten)

https://www.sciencedirect.com/science/article/abs/pii/ S036725300600017X (Artikel Weber \& Sontag 2006, Liste der Publikationen von S. Vogel 1950-2005; kostenpflichtig) https://www.sciencedirect.com/science/article/pii/S0367253 015001565?via\%3Dihub. (Artikel WeBer 2015: Nachruf auf S. Vogel; Liste seiner Publikationen 2006-2015)

\section{Anschriften der Autoren}

Prof. i. R. Dr. Anton Weber, Universität Wien, Department für Botanik und Biodiversitätsforschung, Rennweg 14, A-1030 Wien, E-Mail: anton.weber@univie.ac.at

Dr. Günter Gerlach, Botanischer Garten MünchenNymphenburg, Menzinger Str. 61, 80638 München, E-Mail: gerlach@snsb.de 